\title{
Region- and Cell Type-Specific Facilitation of Synaptic Function at Destination Synapses Induced by Oligodendrocyte Depolarization
}

\author{
당shiko Yamazaki, ${ }^{1}$ Yoshifumi Abe, ${ }^{2}$ Shinsuke Shibata, ${ }^{3}$ Tomoko Shindo, ${ }^{3}$ Satoshi Fujii, ${ }^{1}$ Kazuhiro Ikenaka, ${ }^{4}$ \\ and $\odot$ Kenji F. Tanaka ${ }^{2}$ \\ ${ }^{1}$ Department of Physiology, Yamagata University School of Medicine, Yamagata 990-9585, Japan, ${ }^{2}$ Department of Neuropsychiatry, ${ }^{3}$ Electron Microscope \\ Laboratory, Keio University School of Medicine, Tokyo 160-8582, Japan, and ${ }^{4}$ Division of Neurobiology and Bioinformatics, National Institute for \\ Physiological Sciences, Okazaki 444-8787, Japan
}

The axonal conduction of action potentials affects the absolute time it takes to transmit nerve impulses as well as temporal summation at destination synapses. At the physiological level, oligodendrocyte depolarization facilitates axonal conduction along myelinated fibers in the hippocampus; however, the functional significance of this facilitation is largely unknown. In this study, we examined the physiology of the facilitation of axonal conduction by investigating the changes in synaptic responses at destination synapses using male and female mice in which channelrhodopsin-2 expression was restricted to oligodendrocytes. The subiculum, one of the projection areas of the examined axons at the alveus of the hippocampus, is divided into three regions (proximal, mid, and distal) and contains two types of principal neurons: regular firing and bursting pyramidal cells. We found a significant increase in excitatory synaptic responses following optogenetic oligodendrocyte depolarization in bursting neurons at two of the three regions, but not in regular firing neurons at any region. The long-term potentiation (LTP) induced by theta burst stimulation at the synapses showing a significant increase was also enhanced after oligodendrocyte depolarization. Conversely, the reduction of oligodendrocyte depolarization during theta burst stimulation, which was achieved by photostimulation of archaerhodopsin-T expressed selectively on oligodendrocytes, reduced the magnitude of LTP. These results show that oligodendrocyte depolarization contributes to the fine control of synaptic activity between the axons they myelinate and targets subicular cells in a region- and cell type-specific manner, and suggest that oligodendrocyte depolarization during conditioning of stimuli is involved in the induction of LTP.

Key words: channelrhodopsin-2; hippocampus; long-term potentiation; subiculum

\section{Significance Statement}

All activity in the nervous system depends on the propagation of action potentials. Changes in the axonal conduction of action potentials influence the timing of synaptic transmission and information processing in neural circuits. At the physiological level, oligodendrocyte depolarization facilitates axonal conduction along myelinated fibers. In this study, we investigated the functional significance of the facilitation of axonal conduction induced by physiological oligodendrocyte depolarization. Using optogenetics and electrophysiological recordings, we demonstrated that oligodendrocyte depolarization in mice expressing channelrhodopsin-2 on oligodendrocytes increased excitatory synaptic responses and enhanced the induction of long-term potentiation at destination synapses in a region- and cell type-specific manner. This facilitation may have a hitherto unappreciated influence on the transfer of information between regions in the nervous system.

\section{Introduction}

All activity in the nervous system depends on the propagation of action potentials, which is the principal method of transferring signals from one neuron to the next; therefore, the modulation of axonal conduction influences signal processing in neuronal circuits. The axonal conduction of action potentials is modulated in several different ways, including activity-dependent mechanisms

\footnotetext{
Received June 28, 2018; revised March 1, 2019; accepted March 6, 2019.

Author contributions: Y.Y., S.F., K.I., and K.F.T. designed research; Y.Y., Y.A., S.S., T.S., and K.F.T. performed research; Y.Y. and Y.A. analyzed data; Y.Y., S.S., and K.F.T. wrote the paper.

This work was supported by Japan Society for the Promotion of Science KAKENHI Grants-in-Aid for Scientific Research 25117005 and 16K01943, and a grant from Brain/MINDS by the Japan Agency for Medical Research and Development under Grant JP18dm0207002.
}

The authors declare no competing financial interests.

Correspondence should be addressed to Yoshihiko Yamazaki at yyamazak@med.id.yamagata-u.ac.jp. https://doi.org/10.1523/JNEUROSCI.1619-18.2019

Copyright $\odot 2019$ the authors 
and neurotransmitter-mediated mechanisms. Oligodendrocytes are myelinating glial cells of the CNS that play an essential role in axonal conduction plasticity in myelinated fibers and participate in the fine temporal regulation of neuronal activity in addition to enabling saltatory conduction. Oligodendrocytes respond to axonal activity with depolarization induced by the activation of neurotransmitter receptors (Berger et al., 1992; Ziak et al., 1998; Káradóttir et al., 2005; Yamazaki et al., 2010) and an increase in $\left[\mathrm{K}^{+}\right]_{\mathrm{o}}$ (Yamazaki et al., 2007). We have demonstrated that physiological oligodendrocyte depolarization induces short- and long-term functional plastic changes in axonal conduction along myelinated fibers in hippocampal preparations from mice expressing channelrhodopsin-2 (ChR2) on oligodendrocytes (Yamazaki et al., 2014). The coordination of multiple action potentials and an increase in axonal excitability are involved in these plastic changes, which might underlie the plasticity of white matter that is critical for learning and higher-level cognitive function (Fields, 2010). However, the physiological significance of this facilitation of axonal conduction remains to be elucidated. Since changes in axonal excitability and conduction velocity influence synaptic transmission, we investigated whether oligodendrocyte depolarization affected synaptic responses and the induction of longterm potentiation (LTP), which is fundamental for the storage of information in the brain, between axons myelinated by the examined oligodendrocytes and the target postsynaptic neurons. We also examined whether oligodendrocyte hyperpolarization showed different effects on axonal conduction and synaptic function using mice expressing archaerhodopsin-T (ArchT) exclusively on oligodendrocytes.

\section{Materials and Methods}

Animals and ChR2(C128S)-EYFP and ArchT-EGFP expression on oligodendrocytes. All animal procedures were performed in accordance with the National Institutes of Health Guide for the Care and Use of Laboratory Animals and were approved by the Animal Research Committees of Yamagata University and Keio University. The number of animals used and their suffering were minimized. Animals were housed two to four per cage in the Animal Center of our university on a $12 \mathrm{~h} \mathrm{light/dark} \mathrm{cycle} \mathrm{at}$ constant temperature and humidity with free access to water and rodent food. We generated oligodendrocyte-specific opsin-expressing mice by using a tetracycline-controllable gene induction system (tet system). In this system, double transgenic mice were generated by crossing a proteolipid protein (PLP)-tTA line (Inamura et al., 2012) with a tetO-opsin line (Tanaka et al., 2012; Tsunematsu et al., 2013); tTA protein is expressed under the control of the PLP promoter, and tTA induces opsin gene expression via tTA-tetO tethering. For excitatory opsin expression, PLP-tTA::tetO-ChR2(C128S)-EYFP (enhanced yellow fluorescent protein) double transgenic mice were used (hereafter referred to as PLPChR2). For inhibitory opsin expression, PLP-tTA::tetO-ArchT-EGFP double transgenic mice were used (hereafter referred to as PLP-ArchT). To avoid ArchT-mediated hypomyelination during the developmental period, doxycycline (Dox) was administered through the feeding chow (Dox-containing chow, $100 \mathrm{mg}$ of Dox/kg chow; CLEA Japan). Starting at 2 weeks before slice preparation, normal chow was given to the PLPArchT mice to induce ArchT expression in oligodendrocytes. PLP-ChR2 mice fed a normal diet were used for excitatory optogenetic experiments, except when mentioned otherwise. For the inhibitory optogenetic experiments, a 2 week Dox-off regimen was applied to PLP-ArchT mice to induce ArchT-EGFP after myelination, and such conditioned mice were used in further experiments, except when mentioned otherwise.

Mouse genotyping. The following PCR primer sets were used for mouse genotyping: tetOup ( $5^{\prime}$-AGCAGAGCTCGTTTAGTGAACCGT-3') and intronlow (5'-AAGGCAGGATGATGACCAGGATGT-3') for both tetO-ChR2(C128S)-EYFP and tetO-ArchT-EGFP mice; and PLPU-604 (5' ${ }^{\prime}$-TTTCCCATGGTCTCCCTTGAGCTT-3') and mtTA24L (5'-CG GAGTTGATCACCTTGGACTTGT-3') for PLP-tTA mice. The expected
PCR products were $\sim 610$ and $630 \mathrm{bp}$, respectively. Wild-type mice were negative for the above PCR products.

Immunohistochemistry. The mice were anesthetized deeply and perfused with $4 \%$ paraformaldehyde in $0.1 \mathrm{~m}$ phosphate buffer. Perfused brains were postfixed with the same fixative overnight at $4^{\circ} \mathrm{C}$. After cryoprotection, the brains were embedded in O.C.T. Compound (Sakura Fine Technical Company), frozen, and cut at $40 \mu \mathrm{m}$ thickness. Floating sections in $1 \times$ PBS with $0.1 \%$ Triton X-100 were incubated with the primary antibodies overnight at room temperature. The following antibodies were used: anti-GFP (1:250 dilution; goat polyclonal; catalog \#600-101-215, Rockland; RRID:AB_218182); anti-NeuN (1:500; rabbit monoclonal, clone EPR12763; catalog \#ab177487, Abcam; RRID: AB_2532109); anti-glial fibrillary acidic protein (GFAP; 1:1000; mouse monoclonal, clone GA5; catalog \#G3893, Sigma-Aldrich; RRID: AB_477010); anti-PLP (1:1; rat monoclonal, clone AA3 hybridoma supernatant); and anti-Iba1 (ionized calcium-binding adapter molecule 1; 1:500; rabbit polyclonal; catalog \#019-19741, Wako Chemical; RRID: AB_839504). The sections were incubated with species-specific secondary antibodies conjugated to Alexa Fluor 488 or 568 (1:2000; Invitrogen) for $2 \mathrm{~h}$ at room temperature. Fluorescent images were obtained with a confocal microscope (LSM710, Carl Zeiss).

Transmission electron microscopy analysis. The detailed sample preparation procedure for transmission electron microscopy (TEM) was described previously (Shibata et al., 2015). Briefly, brain slices from each mouse strain were removed after perfusion with $2.5 \%$ glutaraldehyde in saline. The brain slices were postfixed with $2.5 \%$ glutaraldehyde (Electron Microscopy Sciences) in $0.2 \mathrm{M}$ cacodylate buffer, $\mathrm{pH} 7.4$, for $12 \mathrm{~h}$ at $4^{\circ} \mathrm{C}$ and with $1 \% \mathrm{OsO}_{4}$ (Nisshin $\mathrm{EM}$ ) for $2 \mathrm{~h}$ at $4^{\circ} \mathrm{C}$, dehydrated through an ethanol series (50, 70, 80, 90, and 100\% EtOH, acetone, and QY1) twice for $30 \mathrm{~min}$ each, incubated in a graded Epon series (Epon/QY1 = $1: 3,1: 1,3: 1$ ) for $1 \mathrm{~h}$ each and $100 \%$ Epon (Oken) for $96 \mathrm{~h}$ at $4^{\circ} \mathrm{C}$, and embedded in $100 \%$ Epon. After complete polymerization for $72 \mathrm{~h}$ at $60^{\circ} \mathrm{C}$, the resin block was trimmed and cut into $70 \mathrm{~nm}$ ultrathin sections with an ultramicrotome (EM-UC7, Leica). The sections were stained with uranyl acetate and lead citrate for $10 \mathrm{~min}$ each, and were observed under a TEM (JEM 1400plus, JEOL).

Measurements of myelinated axon number, axon diameter, myelin thickness, and g-ratio. ImageJ software (http://rsb.info.nih.gov/ij/) was used for measurements. Optimal brightness and grayscale pixel values were adjusted so as to provide the sharpest discrimination of the myelin/axon border. These traced images were converted into binary images. The number of myelinated axons was counted in six nonoverlapping fields (each field, $100 \mu \mathrm{m}^{2}$ ) of the corpus callosum in each animal. The average from those six fields was considered as the number of myelinated axons/ $100 \mu \mathrm{m}^{2}$ in each animal. For the measurement of axon diameter, myelin thickness, and g-ratio (the ratio of the inner axonal diameter to the total outer diameter), 100 myelinated axons in the corpus callosum were selected randomly in each animal and examined. Axon diameter was defined as the minor axis of an ellipse-approximated axon. The median axon diameter of 100 myelinated axons was considered as the representative value of axon diameter in each animal. The g-ratio was calculated using the equation $0.5 \mathrm{AD} /(0.5 \mathrm{AD}+\mathrm{MT})$, where $\mathrm{AD}$ is the diameter of the axon and MT is myelin thickness. The average myelin thickness and g-ratio of 100 myelinated axons was considered as the representative value of myelin thickness and g-ratio, respectively, in each animal.

Slice preparation. Hippocampal slices were prepared from 41- to 56-dold male or female transgenic mice and male wild-type mice. After the animals were decapitated under deep isoflurane anesthesia, $400-\mu \mathrm{m}$ thick slices were prepared at a $45^{\circ}$ angle to the septotemporal axis using a rotary slicer (DTY-7700, Dosaka) to obtain long alveus fibers in a hippocampal slice and were maintained for at least $1 \mathrm{~h}$ before recording at $30^{\circ} \mathrm{C}$ in artificial CSF (aCSF) containing the following (in mM): $124 \mathrm{NaCl}$, $3 \mathrm{KCl}, 1.25 \mathrm{NaH}_{2} \mathrm{PO}_{4}, 2 \mathrm{MgSO}_{4}, 2.5 \mathrm{CaCl}_{2}, 22 \mathrm{NaHCO}_{3}$, and 10 glucose oxygenated with $95 \% \mathrm{O}_{2} / 5 \% \mathrm{CO}_{2}$.

Electrophysiological recordings. For recordings, a slice was transferred to a recording chamber and perfused continuously with aCSF at a rate of $3 \mathrm{ml} / \mathrm{min}$ at $30^{\circ} \mathrm{C}$. For extracellular field recordings, a stimulating electrode (fine-tip bipolar tungsten electrode) was placed in the alveus of the CA1 region, and compound action potentials (CAPs) were recorded 
from the alveus, close to the subiculum, using glass electrodes filled with aCSF $(8-10 \mathrm{M} \Omega)$ in the presence of the non-NMDA glutamate receptor antagonist 6,7-dinitroquinoxaline-2,3-dione $(20 \mu \mathrm{M})$ to prevent possible contamination by synaptic responses. The CAPs in this region are mainly derived from myelinated axons originating from CA1 pyramidal cells. At the beginning of each experiment, a stimulus-response curve was established by measuring CAP amplitude, and then, the intensity of the stimulus pulse was adjusted to elicit CAPs that were $50-60 \%$ of maximal and maintained at this level throughout the experiment. Baseline responses were recorded following the delivery of the test stimuli every $15 \mathrm{~s}$. After stable responses were obtained for $>10 \mathrm{~min}$, a short pulse (5-500 ms) of blue light (high-power blue LED, $470 \mathrm{~nm}$ ) or yellow light (high-pressure mercury lamp with Texas Red filter; duration, $30 \mathrm{~s}$ ) was delivered to activate the ChR2 or ArchT channels expressed on oligodendrocytes, respectively. To evaluate changes in CAPs induced by oligodendrocyte depolarization, their amplitude, width, and area were measured at 1-3 and $28-30 \mathrm{~min}$ after photostimulation and were expressed as a ratio of the mean responses recorded during the $8 \mathrm{~min}$ period before the delivery of the light pulse. For whole-cell recordings, pyramidal cells in the subiculum and CA1 region and oligodendrocytes in the CA1 region were visualized using an infrared differential interference contrast (IR-DIC) microscope (E600-FN, Nikon) with a $40 \times$ water-immersion objective. Patch electrodes were pulled from borosilicate glass (World Precision Instruments) using a micropipette puller (P-97, Sutter Instrument). The electrodes were filled with a solution containing the following (in $\mathrm{mM}$ ): 140 K-gluconate, 10 HEPES, 0.5 EGTA, $10 \mathrm{NaCl}, 1 \mathrm{MgCl}_{2}, 2 \mathrm{Mg}$-ATP, and $0.2 \mathrm{Na}-\mathrm{GTP}$, adjusted to $\mathrm{pH} 7.3$ with $\mathrm{KOH}$. The resistance of the electrodes was set to 5-7 and 8-10 M $\Omega$ for pyramidal cells and oligodendrocytes, respectively. Whole-cell recordings were performed from the soma in either voltage-clamp or current-clamp mode at near resting membrane potential $(-60$ to $-70 \mathrm{mV})$. EPSCs were evoked by stimulation of the alveus between the CA1 region and subiculum, where the axons from CA1 pyramidal cells pass through to reach their target subicular neurons, and recorded from voltage-clamped subicular neurons in each region once every $15 \mathrm{~s}$. The division of the subiculum into three equal regions (proximal, mid, and distal) was made by visual inspection of the subiculum between the proximal end (where the condensed pyramidal cell layer of CA1 begins to broaden into the thicker layer of the subiculum) and the distal end (border with the presubiculum). Series resistance was monitored throughout the experiments by the application of hyperpolarizing pulses through the patch pipette; if series resistance changed by $>25 \%$, the experiment was stopped and the data were discarded. The amplitude of EPSCs was measured to evaluate the changes in excitatory synaptic transmission. After EPSC amplitude had stabilized, a short pulse $(500 \mathrm{~ms})$ of blue or yellow light $(30 \mathrm{~s})$ was applied. The mean amplitude during the 8 or 10 min period immediately before the delivery of light illumination was defined as the $100 \%$ level. The mean amplitude at $1-3$ and $28-30 \mathrm{~min}$ after photostimulation was calculated to evaluate the magnitude of the change in EPSCs induced by oligodendrocyte depolarization. To calculate the paired-pulse ratio (PPR), the first EPSCs were subtracted from the current responses evoked with paired-pulse stimulation (interstimulus interval, $50 \mathrm{~ms}$ ). By this procedure, the second EPSCs were isolated and the PPR could be calculated accurately. The mean PPR during the 6 min period immediately before the delivery of light illumination was defined as the $100 \%$ level. Antidromic action potentials were recorded from CAl pyramidal cells by stimulating the axons of the recorded pyramidal cells, which were toward the subiculum, and latency was measured as the time from the artifact of electrical stimulation to the start of the action potential. The start of the action potential was taken as the time when the slope of the tangent to the action potential became $>10 \mathrm{mV} / \mathrm{ms}$. The action potentials induced by antidromic stimulation were recorded once every $15 \mathrm{~s}$. The mean latency during the $3 \mathrm{~min}$ period immediately before the delivery of blue light was defined as the $100 \%$ level. The mean latency at 1-3 min after photostimulation was calculated to evaluate the change in axonal conduction induced by oligodendrocyte depolarization. For the LTP experiments, stimulation intensity was set to produce $40-60 \%$ of the maximum response without an action current, and theta burst stimulation $(5,10,15$, or 20 bursts, with each burst containing four pulses at $100 \mathrm{~Hz}$ and individual bursts sepa- rated by $200 \mathrm{~ms}$ ) was delivered without or at $3 \mathrm{~min}$ after $500 \mathrm{~ms}$ blue light illumination to induce LTP. The mean amplitude during the $10 \mathrm{~min}$ period immediately before the delivery of blue light was defined as the $100 \%$ level. The mean amplitude at 35-40 min after theta burst stimulation (at 38-43 min after blue light illumination in the photostimulation experiments) was calculated to evaluate the magnitude of LTP. In the LTP experiments using mice expressing ArchT on oligodendrocytes, yellow light illumination was initiated at $5 \mathrm{~s}$ before first burst stimulation and terminated at $25 \mathrm{~s}$ after the end of the last burst. Potential and current responses were recorded using Axopatch-200B or Axopatch-1D amplifiers (Molecular Devices), filtered $(5 \mathrm{kHz})$, and stored in a computer after conversion (digitized at $50-100 \mathrm{kHz}$ ) by an analog-to-digital converter (PCI-6023E, National Instruments). Data were analyzed off-line using a wave analysis program developed by ourselves and OriginLab. Drugs were obtained from Sigma-Aldrich and were bath applied.

Biocytin staining. To stain the recorded pyramidal cells, the patch electrodes were filled with $0.5 \%$ biocytin. After recording was completed, the patch electrode was withdrawn carefully, and the slice was removed from the recording chamber and fixed overnight at room temperature in a solution of $4 \%$ paraformaldehyde in sodium phosphate buffer, $\mathrm{pH}$ 7.4. The fixed slice was then rinsed in PBS, pH 7.4, treated for 30 min with $1 \%$ $\mathrm{H}_{2} \mathrm{O}_{2}$ to neutralize endogenous peroxidase, and then, following several rinses in PBS, incubated for $24 \mathrm{~h}$ at room temperature with avidinbiotinylated peroxidase complex diluted in PBS containing 0.3\% Triton $\mathrm{X}-100$. Finally, following a $30 \mathrm{~min}$ rinse in PBS, the slice was incubated with $0.06 \% \mathrm{DAB}$ and $0.006 \% \mathrm{H}_{2} \mathrm{O}_{2}$, diluted in PBS.

Experimental design and statistical analysis. All data are expressed as the mean \pm SEM. Sample size $n$ refers to the number of neurons, oligodendrocytes, or hippocampal slices analyzed in electrophysiological recordings. In TEM analysis, sample size $n$ refers to the number of animals. As the initial data obtained from electrophysiological recordings were indistinguishable between male and female mice, all data were combined for statistical analysis. For statistical analysis of the input-output relationship between wild-type mice and PLP-ChR2 or PLP-ArchT mice, twoway repeated-measures ANOVA was used. Significant changes in the amplitude, width, and area of CAPs in PLP-ChR2 mice (no photostimulation vs photostimulation) were assessed using an unpaired two-tailed Student's $t$ test. Significant changes in the amplitude of EPSCs, latencies of antidromic action potentials, and PPR (before vs after photostimulation at the indicated period) were assessed using a paired two-tailed Student's $t$ test. For the statistical analysis of the magnitude of LTP in PLP-ChR2 mice, an unpaired two-tailed Student's $t$ test was used to compare the data between the control group in which theta burst stimulation was not delivered and the groups in which theta burst stimulation was applied, as indicated by the number of bursts. For statistical analysis of the magnitude of LTP in PLP-ArchT mice, two-way ANOVA with Tukey's post hoc test was used to compare the data generated in the presence and absence of yellow light illumination, and between PLPArchT mice in the presence of yellow light illumination and PLP-ArchT mice with Dox treatment in the presence of yellow light illumination. For the statistical analysis of the myelinated axon number, axon diameter, myelin thickness, and g-ratio, an unpaired two-tailed Student's $t$ test was used to compare the data between the control mice and PLP-ChR2 or PLP-ArchT mice. A difference was considered statistically significant at $p<0.05$.

\section{Results}

\section{Oligodendrocyte depolarization facilitates synaptic} transmission at destination synapses

Oligodendrocytes in the alveus form a myelin sheath for the axons of CA1 pyramidal cells. A single axon from a pyramidal cell soma projects through the stratum oriens and into the alveus. Typical CA1 axons branch at the alveus and arrive at several targets both within and beyond the hippocampus. The subiculum is adjacent to the CA1 region and has significant connections with CA1 neurons. Therefore, we focused on the synapses between CAl neurons and subiculum neurons to investigate the functional significance of the facilitation of axonal conduction 
A

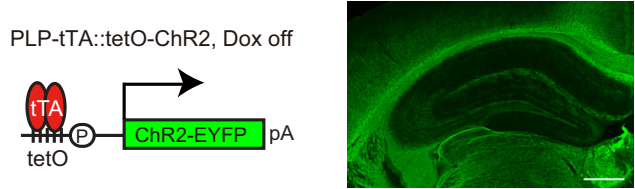

B
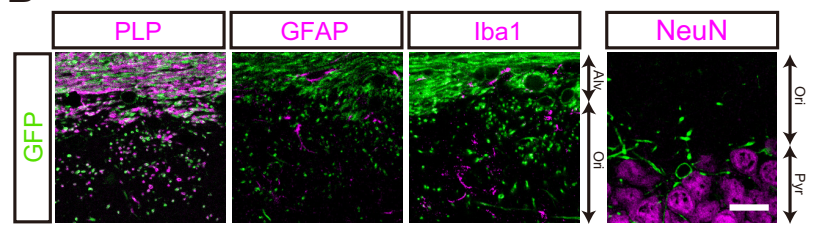

C

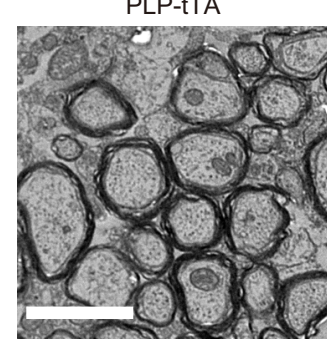

PLP-tTA::tetO-ChR2

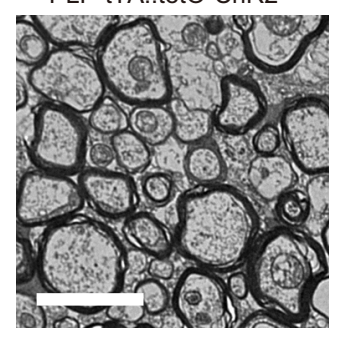

D

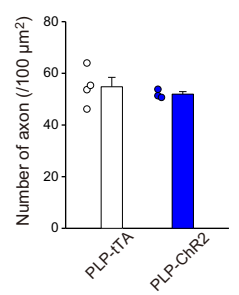

E

$\mathbf{F}$

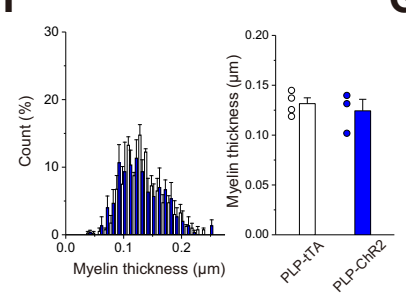

G

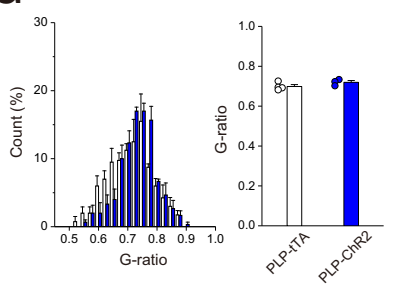

H

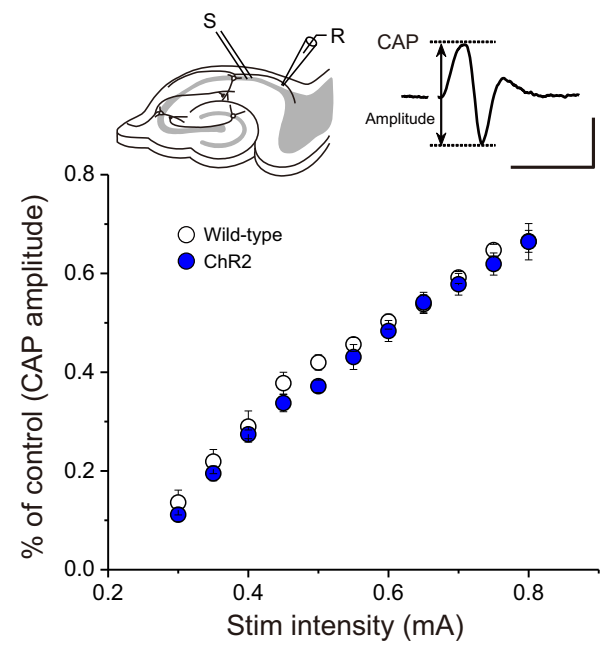

Figure 1. Characterization of PLP-ChR2 mice. A, Induction of oligodendrocyte-specific ChR2(C128S)-EYFP expression in the tet system (left). tTA, Tetracycline-controlled transcriptional activator; tet0, Tetracycline-controlled transcriptional activator-dependent promoter. induced by oligodendrocyte depolarization. Previously, the effects of oligodendrocyte depolarization on axonal conduction were mainly studied in the axons of CA1 pyramidal cells projecting toward the fimbria (Yamazaki et al., 2014), but not toward the subiculum. Therefore, at first, we recorded CAPs that were evoked in the CA1 region and propagated in the subiculum, and measured the changes in CAP induced by oligodendrocyte depolarization in PLP-ChR2 mice (Fig. 1A). As shown previously (Yamazaki et al., 2014), EYFP expression patterns were similar to the localization of myelin in PLP-ChR2 mice. EYFP-positive fibrous structures were colabeled with PLP, but none were colabeled with NeuN (neuron marker), GFAP (astrocyte marker), or Ibal (microglia marker) in the hippocampal alveus of PLP-ChR2 mice (Fig. 1B). In TEM analysis, the number of myelinated fibers, axon diameter, myelin thickness, and g-ratio in PLP-ChR2 mice $(n=$ 3 ) were comparable to those in control mice $(n=4)$ at 4 weeks of age (myelinated axon number: $t_{(5)}=0.65, p=0.50$; axon diameter: $t_{(5)}=0.84, p=0.44$; myelin thickness: $t_{(5)}=0.61, p=0.62$; g-ratio: $t_{(5)}=1.54, p=0.17$; Fig. $1 C-G$ ), indicating that oligodendrocytic ChR2-EYFP expression during the developmental period did not affect myelination in PLP-ChR2 mice. Input-output curves generated by plotting the amplitude of CAPs versus stimulation strength were not significantly different between wild-type mice $(n=4)$ and PLP-ChR2 mice $\left(n=4 ; F_{(1,60)}=2.16\right.$, $p=0.19$, two-way repeated-measures ANOVA; Fig. $1 H)$. These results show that axonal excitability and the number of axons activated at each stimulus intensity were not influenced by ChR2 expression on oligodendrocytes. When blue light was applied for 5,10 , or $25 \mathrm{~ms}$, there was no significant change in CAP amplitude at 1-3 min (early phase) or 28-30 min (late phase) after photostimulation (Fig. $2 A, B$ ). Using a $50 \mathrm{~ms}$ pulse, CAP amplitude increased at the late phase $(115.0 \pm 3.6 \%$ of baseline, $n=8$; $t_{(12)}=2.95, p=0.012$ vs no photostimulation), but not at the early phase $\left(104.8 \pm 1.7 \%\right.$ of baseline; $n=8, t_{(12)}=1.91, p=$ 0.080 vs no photostimulation; Fig. $2 A, B)$. In the case of a $500 \mathrm{~ms}$ pulse, CAP amplitude showed a significant increase at both the early phase $\left(108.4 \pm 1.8 \%\right.$ of baseline; $n=9, t_{(13)}=3.66, p=$ 0.0029 , vs no photostimulation) and the late phase (126.8 $\pm 5.5 \%$ of baseline; $n=9, t_{(13)}=3.59, p=0.0033$, vs no photostimulation; Fig. $2 A, B)$. As the duration of blue light illumination increased, CAP amplitude increased. Moreover, for a $500 \mathrm{~ms}$ pulse, a transient decrease in CAP width was observed at 1-3 min after photostimulation $\left(92.2 \pm 1.4 \%\right.$ of baseline; $n=9, t_{(13)}=4.22$, $p=0.0010$ vs no photostimulation). Its width gradually returned to baseline after $\sim 10 \mathrm{~min}$, with the result that, at $28-30 \mathrm{~min}$ after stimulation, no significant change in CAP width was seen $\left(101.1 \pm 0.8 \%\right.$ of baseline; $n=9, t_{(13)}=1.65, p=0.12$ vs no photostimulation; Fig. 2 C). Conversely, CAP area showed a sig-

$\leftarrow$

Fluorescent image of the hippocampus labeled with GFP antibody (right). Scale bar, $500 \mu \mathrm{m} . \boldsymbol{B}$ Double immunohistochemistry showing that PLP-positive myelin fibers were labeled with GFP $(60.1 \pm 2.2 \% ; 4$ sections, 2 animals). None of the GFAP-, Iba1-, or NeuN-positive signals were colabeled with the anti-GFP antibody. Purple, Marker; green, GFP; Alv, alveus; Ori, stratum oriens; Pyr, CA1 pyramidal cell layer in the hippocampus. Scale bar, $10 \mu \mathrm{m}$. C, Transmission electron micrographs from control mice (PLP-tTA mice, left) and PLP-ChR2 mice (right) at 4 weeks of age, showing normal-appearing myelin structure in the corpus callosum of PLP-ChR2 mice. Scale bar, $1 \mu \mathrm{m}$. D, The number of myelinated axons in the corpus callosum of PLP-ChR2 mice $(n=3)$ and control mice $(n=4)$. $\boldsymbol{E}-\boldsymbol{G}$, Histograms (left) and group data (right) of axon diameter $(\boldsymbol{E})$, myelin thickness $(\boldsymbol{F})$, and g-ratio $(\boldsymbol{G})$ of PLP-ChR2 mice $(n=3)$ and control mice $(n=4)$. $\boldsymbol{H}$, Schematic drawing showing the stimulating $(S)$ and recording (R) electrodes for recording CAPs (top, left). Measurement of the amplitude of CAPs (top, right). Calibration: $5 \mathrm{ms,}$ $0.5 \mathrm{mV}$. Input- output relationship of CAP amplitude in wild-type and PLP-ChR2 mice (bottom). 

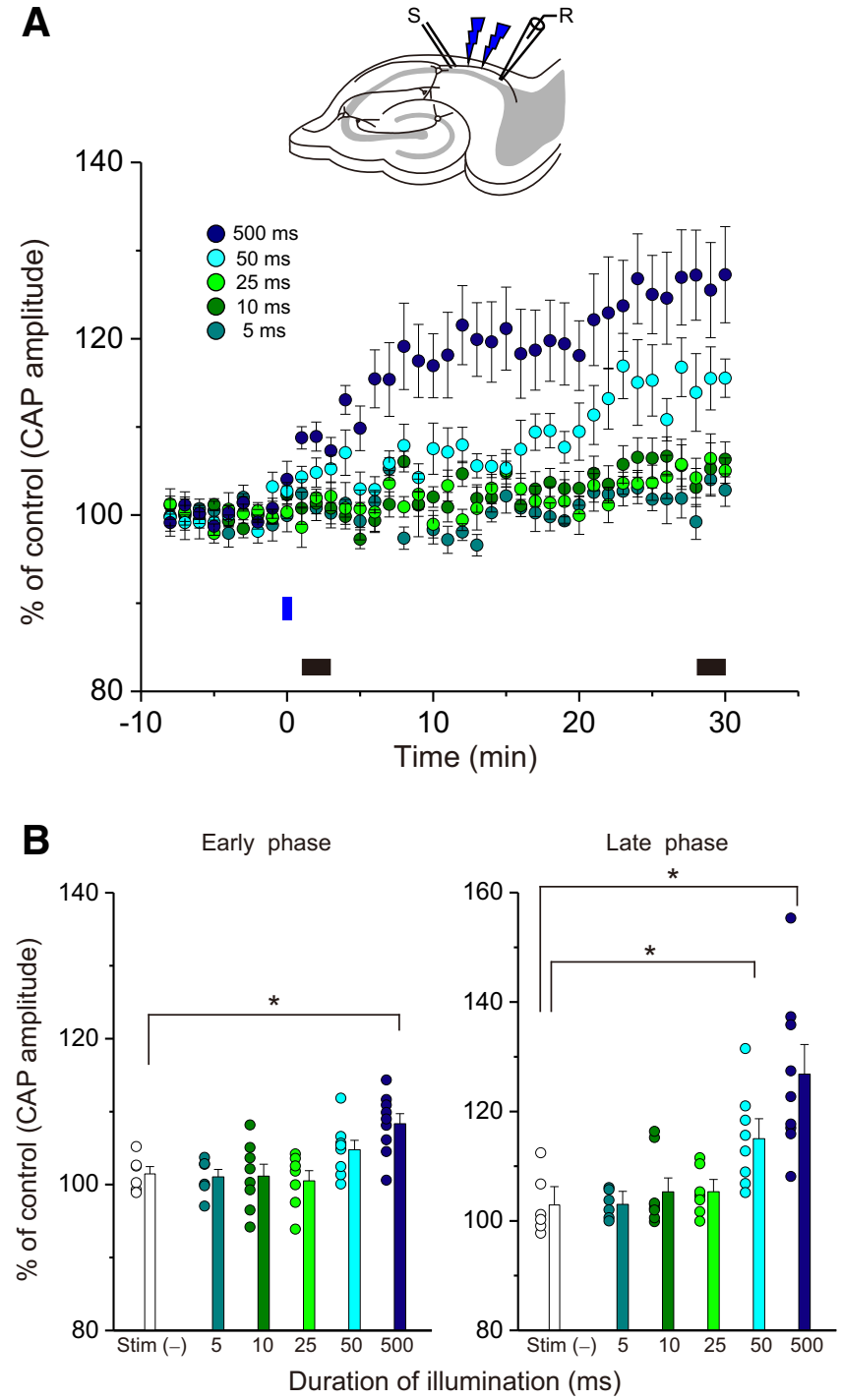
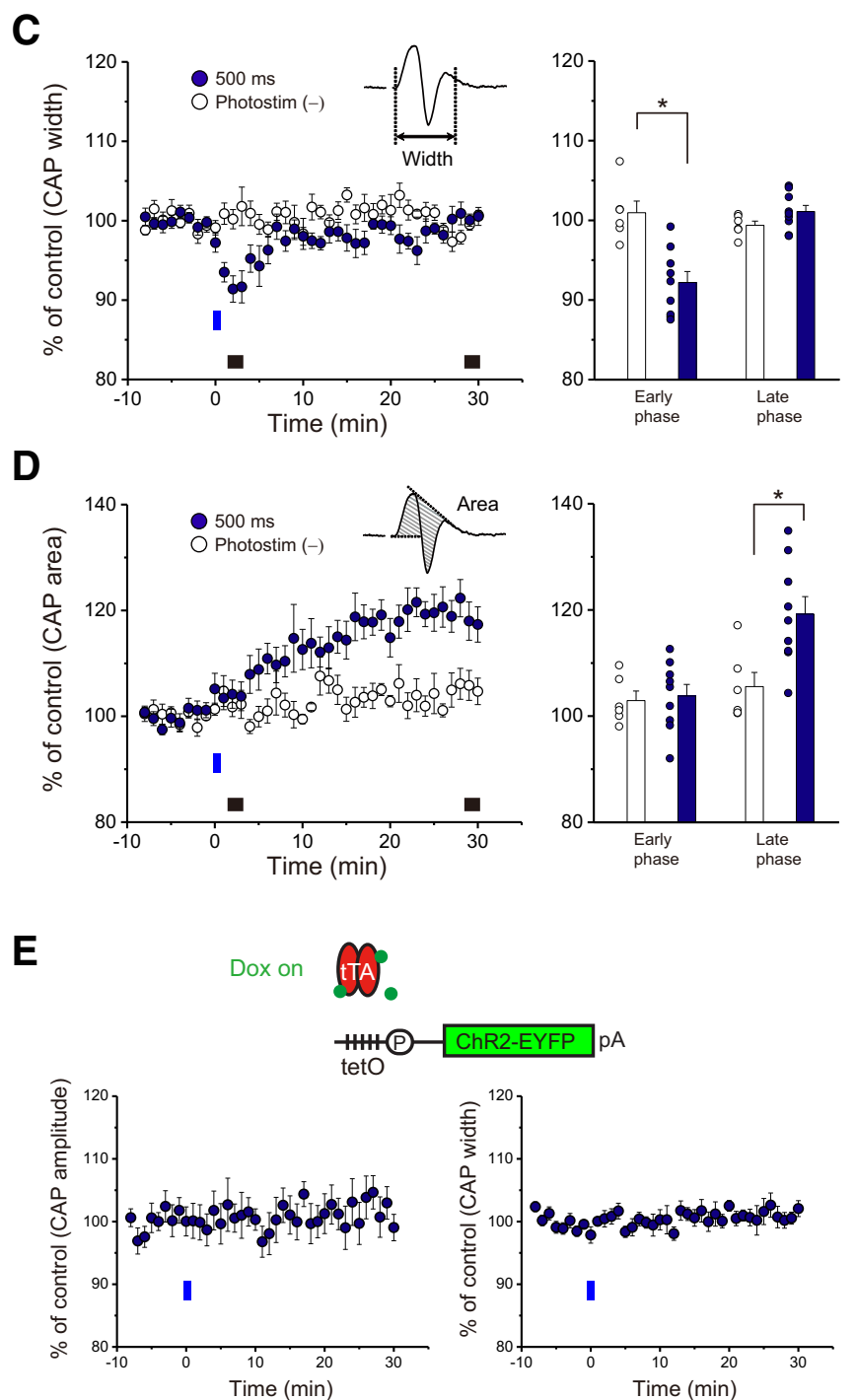

Figure 2. Depolarization of oligodendrocytes in the alveus facilitates axonal conduction along the axons toward the subiculum. $\boldsymbol{A}$, Relationship between the duration of illumination for oligodendrocyte depolarization and the change in CAP amplitude of the axons toward the subiculum. Changes in CAP amplitude induced by blue light photostimulation for $5 \mathrm{~ms}(n=6), 10 \mathrm{~ms}(n=$ 8), $25 \mathrm{~ms}(n=7), 50 \mathrm{~ms}(n=8)$, or $500 \mathrm{~ms}(n=9)$, starting at $0 \mathrm{~min}$, expressed as a percentage of mean CAP amplitude during the 8 min period before light pulse delivery. The blue bars indicate the timing of blue light illumination. The black bars indicate the measurement periods in $(\boldsymbol{B})$. Inset, Schematic drawing showing the stimulating $(S)$ and recording $(R)$ electrodes for recording $(A P s$. $B$, Summary histograms for the change in CAP amplitude at 1-3 $\mathrm{min}$ (left) or 28-30 min (right) after photostimulation for the indicated time, expressed as a percentage of the mean CAP amplitude during the 8 min period before light pulse delivery. Stim, Stimulation. ${ }^{*} p<0.05$. C, Left, Changes in CAP width over time with no photostimulation $(n=6)$ or after $500 \mathrm{~ms}$ photostimulation $(n=$ 9). Inset is a measurement of CAP width. Right, Summarized results for the changes in CAP width. $\boldsymbol{D}$, Left, Changes in CAP area over time with no photostimulation ( $n=6$ ) or after $500 \mathrm{~ms}$ photostimulation $(n=9)$. Inset is a measurement of CAP area. Right, Summarized results for the changes in CAP area. $E$, Effects of blue light illumination on the amplitude (left) and width (right) of CAP in mice with the same genotype as PLP-ChR2 mice, but expression was blocked by Dox administration.

nificant increase at $28-30$ min after stimulation $(119.2 \pm 3.3 \%$ of baseline; $n=9, t_{(13)}=2.97, p=0.0011$ vs no photostimulation), but not at $1-3 \mathrm{~min}$ after stimulation ( $103.8 \pm 2.2 \%$ of baseline; $n=9, t_{(13)}=0.29, p=0.78$ vs no photostimulation; Fig. $\left.2 D\right)$. These results indicate the synchronization of action potentials in the early phase, but not in the late phase, consistent with the results of a previous study (Yamazaki et al., 2014). We also performed control experiments in mice with the same genotype as the PLP-ChR2 mice, but expression was blocked by Dox treatment to confirm that these modulations were induced by oligodendrocyte activation rather than the effects of photostimulation alone. Photostimulation induced neither significant depolarization of oligodendrocytes $(0.24 \pm 0.03 \mathrm{mV}$ shift; $n=4)$ nor a significant effect on CAPs in PLP-ChR2 mice with Dox treatment (Fig. 2E). Thus, oligodendrocyte depolarization facilitates axonal conduction in axons projecting toward the subiculum, as seen in axons projecting toward the fimbria.

Then, we investigated the effects of oligodendrocyte depolarization on the synaptic responses between axons myelinated by the examined oligodendrocytes and subicular neurons. Two types of pyramidal cells have been characterized in the subiculum according to their firing properties (i.e., regular firing cells and bursting cells; Wozny et al., 2008b). We first examined whether both types of subicular pyramidal cells were included in the mice with ChR2-expressing oligodendrocytes by using whole-cell recordings from subicular neurons (Fig. $3 A$ ). Consistent with previous studies, a subpopulation of subicular pyramidal cells showed a regular firing pattern with some frequency adaptation and amplitude accommodation (Fig. 3B). Another subpopulation of subicular pyramidal cells showed bursting firing. 
A
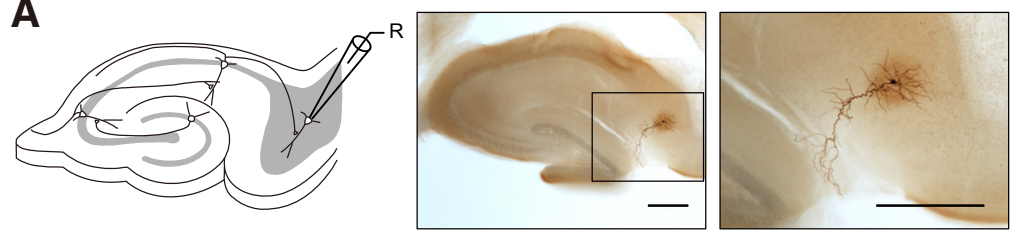

B

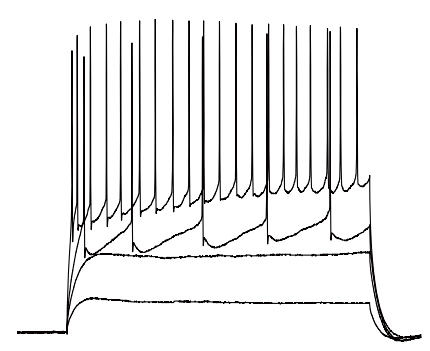

D

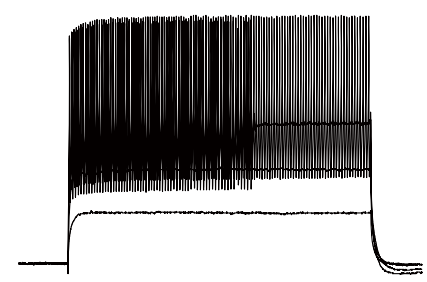

C

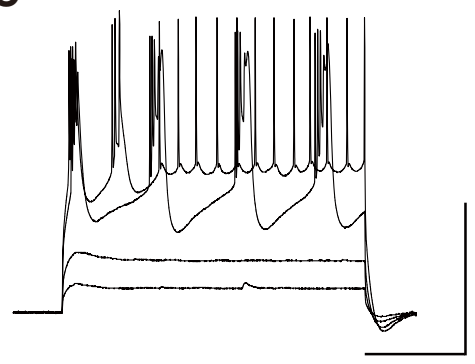

$\mathbf{E}$

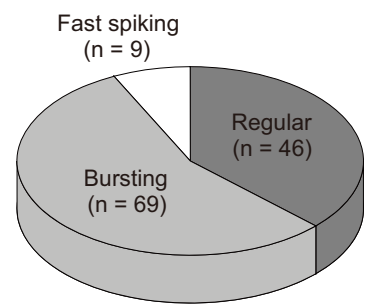

Figure 3. Firing properties of subicular pyramidal cells. $A$, Schematic drawing showing the recording pipette for whole-cell recording from a subicular pyramidal cell (left). Light microscopy showing a biocytin-stained pyramidal cell at low (middle) and high (right) magnification. Scale bar, $200 \mu \mathrm{m}$. $\boldsymbol{B}-\boldsymbol{D}$, Voltage responses to intracellularly injected current pulses in a regular firing neuron $(\boldsymbol{B})$, a bursting neuron $(\boldsymbol{C}$ ), and a fast-spiking interneuron (D). Calibration: $200 \mathrm{~ms}, 50 \mathrm{mV}$. $\boldsymbol{E}$, Pie chart for the ratio of subicular neurons showing each firing pattern.

Table 1. Summary of the number (ratio) of neurons showing each firing pattern in the subregions of the subiculum

\begin{tabular}{llll}
\hline & Regular firing & Bursting & Fast spiking \\
\hline Proximal & $19(45.2 \%)$ & $20(47.6 \%)$ & $3(7.1 \%)$ \\
Mid & $13(33.3 \%)$ & $24(61.5 \%)$ & $2(5.1 \%)$ \\
Distal & $14(32.6 \%)$ & $25(58.1 \%)$ & $4(9.3 \%)$ \\
Total & $46(37.1 \%)$ & $69(55.6 \%)$ & $9(7.3 \%)$ \\
\hline
\end{tabular}

The ratio of neurons exhibiting each firing pattern was not homogenous in each region of the subiculum, but all types of neurons were observed in all regions.

These bursts occurred at an interval of $\sim 200 \mathrm{~ms}$, consistent with the theta rhythm (Fig. 3C). In the cells showing a pyramidal shape under IR-DIC observation, a small number exhibited considerably high-frequency firing (Fig. 3D). The distribution of the dendrites of these cells was clearly different from that of pyramidal cells, and they were considered to be interneurons (data not shown). From 124 recordings of subicular neurons, $55.6 \%$ of the examined neurons were bursting cells, $37.1 \%$ were regular firing cells, and $7.3 \%$ were fast-spiking interneurons (Fig. 3E). Although the ratio of pyramidal cells exhibiting each firing pattern was not homogenous in each region of the subiculum, as reported previously (Jarsky et al., 2008), both types of pyramidal cells were observed in all regions (Table 1). Projections from CA1 to the subiculum are organized topographically (Amaral et al., 1991), as follows: proximally located CA1 cells project to the distal region of the subiculum (Fig. 4A, red triangle and oval); distally located CA1 cells project just across the border into the proximal region

of the subiculum (Fig. 4A, green triangle and oval); and CA1 cells located in the mid region project to the mid region of the subiculum (Fig. 4A, yellow triangle and oval). Therefore, we examined the effects of oligodendrocyte depolarization induced by photostimulation on the EPSCs recorded from subicular cells in each region and their firing patterns separately (Fig. 4B). EPSCs were evoked by electrical stimulation to the axons of CA1 pyramidal cells, and their amplitudes were measured to evaluate the changes in EPSCs once every $15 \mathrm{~s}$ (Fig. $4 B$ ). In accordance with the evaluation of CAP changes, the changes in EPSC amplitude were evaluated in both phases by measuring the mean value at $1-3 \mathrm{~min}$ and $28-30 \mathrm{~min}$ after photostimulation for the early and late phase, respectively. As shown in Figure 4, $C$ and $E$, for regular firing cells, oligodendrocyte depolarization did not influence synaptic responses at the destination synapses in any region of the subiculum at both phases. Conversely, EPSCs recorded from the bursting cells located in the mid and distal regions, but not in the proximal region, showed a significant increase in their amplitude at both phases (mid-early: $109.7 \pm 5.4 \%$ of baseline; $n=$ $8, t_{(7)}=2.43, p=0.046$; mid-late: $109.8 \pm$ $4.3 \%$ of baseline; $n=8, t_{(7)}=3.02, p=$ 0.019 ; distal-early: $110.3 \pm 4.3 \%$ of baseline; $n=11, t_{(10)}=2.97, p=0.014$; distallate: $112.3 \pm 3.7 \%$ of baseline; $n=11, t_{(10)}$ $=4.97, p=0.0056$; Fig. $4 D, E)$. These results suggest that the modulatory effects of oligodendrocyte depolarization on the responses of destination synapses depended on the cell type and the region of the subiculum.

To address the region specificity of the effects on synaptic transmission, we investigated whether the effects of oligodendrocyte depolarization on axonal conduction along the axons toward the subiculum are different between the axons projecting to different regions (Fig. 5A). The latency of antidromicconducted action potentials recorded from CA1 pyramidal cells close to the $\mathrm{CA} 2$ region, whose axons provide input to neurons in the distal region of the subiculum, was significantly decreased by oligodendrocyte depolarization with photostimulation (1-3 min after photostimulation: $96.3 \pm 1.7 \%$ of baseline; $n=9, t_{(8)}=$ $2.46, p=0.039$; Fig. $5 B$ ), as seen in the axons toward the fimbria (Yamazaki et al., 2014). Conversely, oligodendrocyte depolarization had no significant effect on the axons originating from CA1 close to the subiculum, which provide input to neurons in the proximal region of the subiculum ( $98.9 \pm 0.7 \%$ of baseline; $n=$ $9, t_{(8)}=1.58, p=0.15$; Fig. $5 C$ ). The absence of an effect of oligodendrocyte depolarization in the axons of pyramidal cells located close to the subiculum would account for the lack of modulation at the synapses between the axons of distal CA1 neurons and proximal subicular neurons.

To address the characteristics of the increases in EPSCs, we investigated the changes in the PPR of EPSCs induced by oligodendrocyte depolarization in the mid and distal regions of the subiculum, which showed an increase of EPSC amplitude follow- 
A

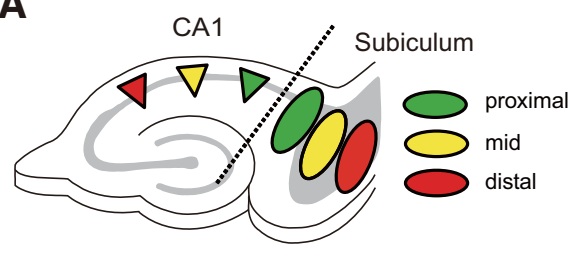

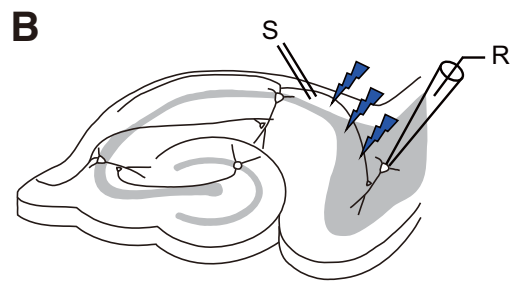

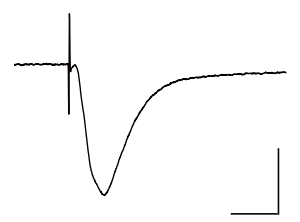

C Regular

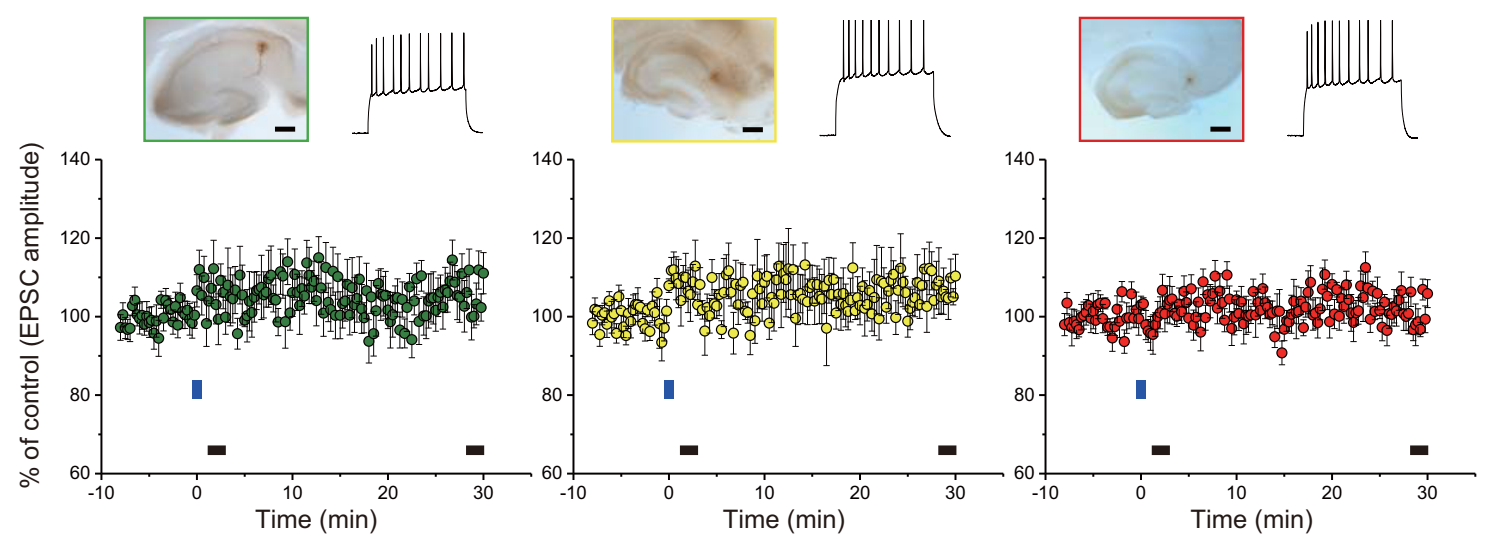

D Bursting

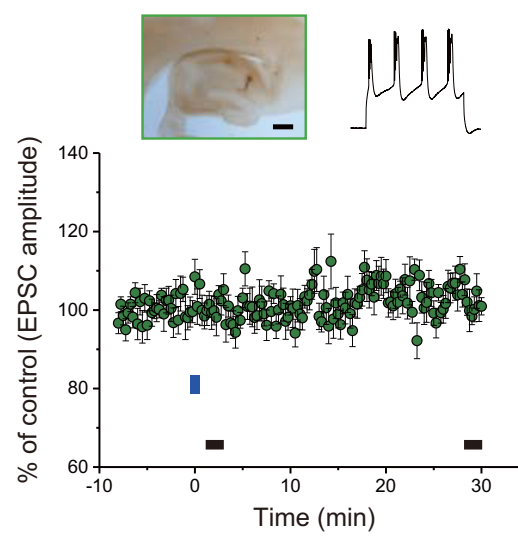

$\mathbf{E}$

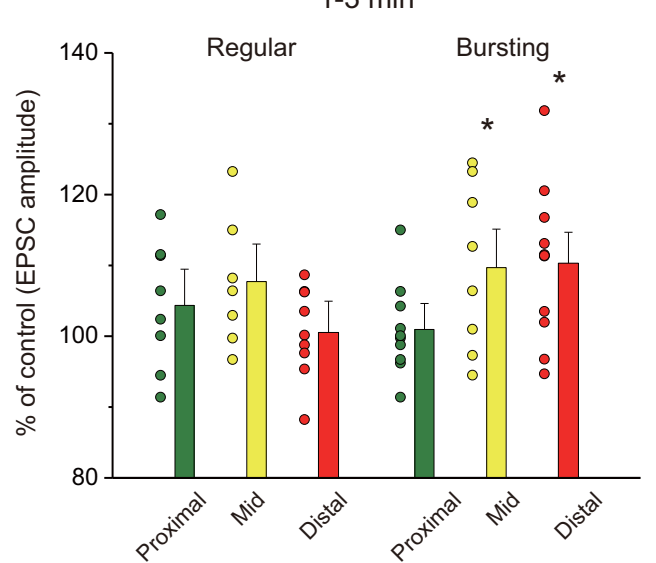

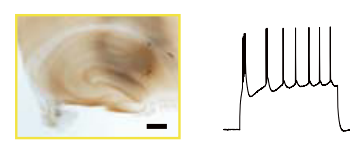
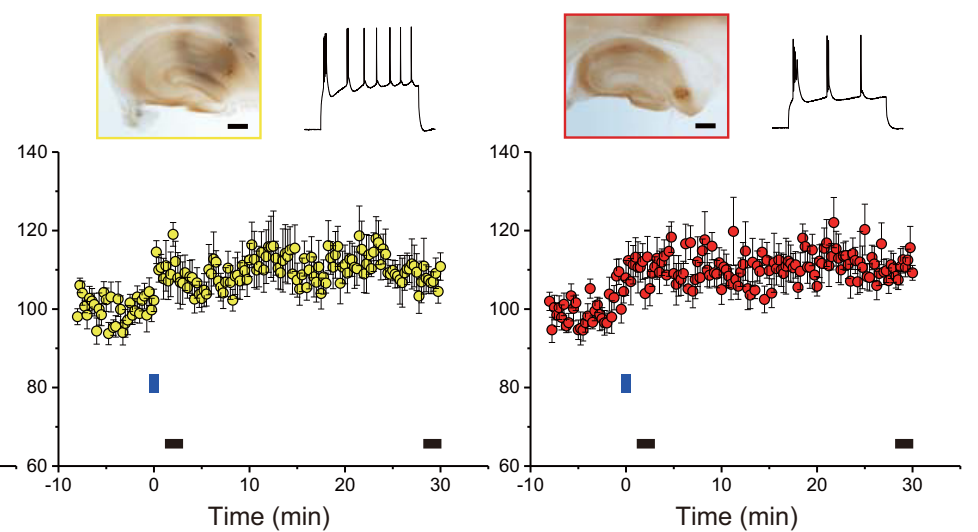

28-30 min

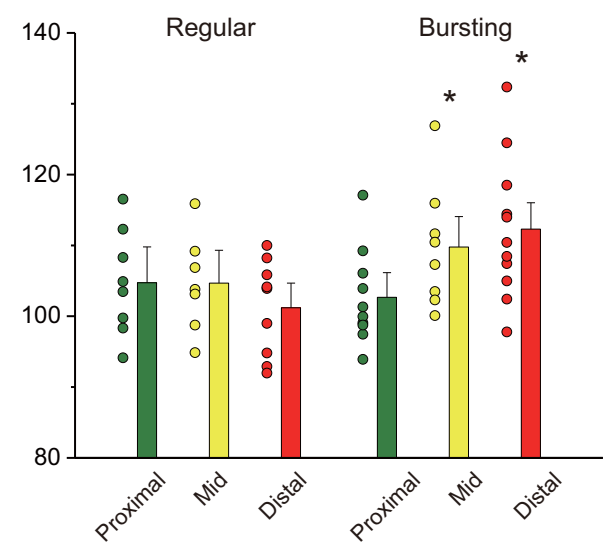

Figure 4. Effects of oligodendrocyte depolarization on synaptic transmission at destination synapses. $\boldsymbol{A}$, Topographic projections from the CA1 region to the subiculum. The pyramidal cells in each portion of the CA1 region indicated by the colored triangles project their axons to each region indicated by an oval with the same color in the subiculum. $B, S$ Shematic drawing showing the stimulating (S) and recording (R) electrodes for recording EPSCs from subicular neurons (left) and a typical EPSC waveform (right). Calibration: $50 \mathrm{~ms}, 100 \mathrm{pA}$. C, D, Time course of the changes in EPSCS induced by oligodendrocyte depolarization in regular firing neurons $(\boldsymbol{C})$ and bursting neurons $(\boldsymbol{D})$ in the proximal (green, $n=8$ and $n=10$ ),(Figure legend continues.) 

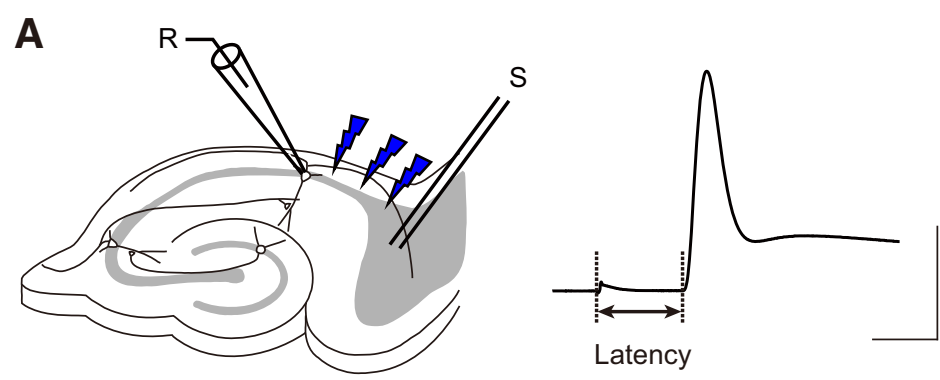

B
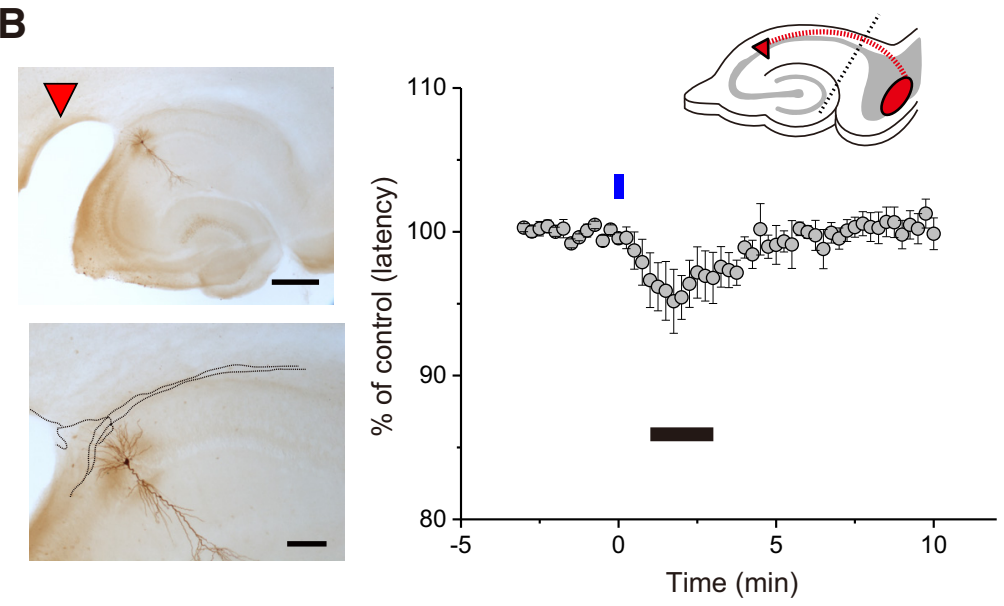

C
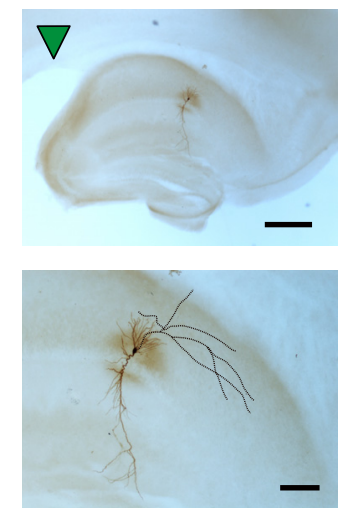$$
\text { (2) }
$$

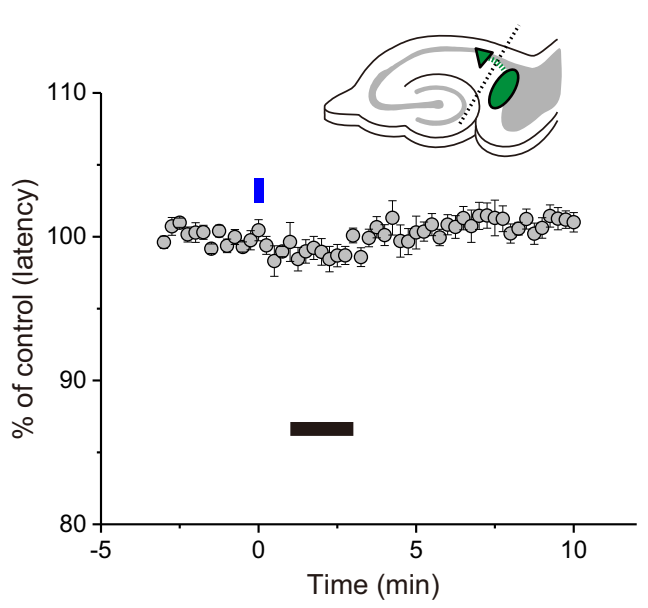

Figure 5. Effects of oligodendrocyte depolarization on the conduction latency of CA1 pyramidal cell axons. $A$, Left, Schematic drawing showing the stimulating $(S)$ and recording ( $R$ ) electrodes for recording antidromic action potentials. Right, Measurement of the conduction latency of the antidromic action potential. Calibration: $2 \mathrm{~ms}, 50 \mathrm{mV} . \boldsymbol{B}, \boldsymbol{C}$, Time course of the latency of action potentials plotted every $15 \mathrm{~s}$ after $500 \mathrm{~ms}$ of photostimulation, expressed as a percentage of the mean value of the action potential latency recorded during the 3 min period before light pulse delivery in proximally $(\boldsymbol{B})$ and distally $(\boldsymbol{C})$ located CA1 pyramidal cells. Insets are schematic drawings showing the position of the pyramidal cell. Left, Typical examples of biocytin-stained pyramidal cells. The axons are traced with dotted lines. Scale bar, $500 \mu \mathrm{m}$ (top) and $200 \mu \mathrm{m}$ (bottom). ing oligodendrocyte depolarization (Fig. $6 A)$. Since the magnitude of the increase in EPSC amplitude recorded from bursting cells at the mid region was not different from that at the distal region, the data from these two regions were combined. Usually, the second EPSC is larger than the first when paired stimuli are applied at an interval of $50 \mathrm{~ms}$ (Fig. 6A). If the increase of EPSCs is due to the facilitation of glutamate release from presynaptic terminals, the PPR will decrease, while this ratio would not change when the increase of EPSCs is solely due to a postsynaptic mechanism. While the amplitude of EPSCs increased at both the early and late phases (Fig. 4D,E), the PPR was increased only at the late phase (1-3 min after photostimulation: $105.2 \pm 6.4 \%$ of baseline; $n=11, t_{(10)}=1.88, p=0.090 ; 28-30 \mathrm{~min}$ after photostimulation: $131.4 \pm 7.8 \%$ of baseline; $n=11, t_{(10)}=4.54, p=0.0011$; Fig. $6 B, D)$, indicating that the enhancement of synaptic transmission by oligodendrocyte depolarization could consist of two different mechanisms. We confirmed that the PPR obtained from regular firing cells was not changed by oligodendrocyte depolarization (Fig. $6 C)$. Since both the amplitude and PPR of EPSCs increased at the late phase in bursting neurons, it is considered that (1) there is a decrease in glutamate release and an increase in the postsynaptic mechanism, but the effect of the latter is larger than that of the former; and (2) another mechanism, which cannot be described using a conventional presynaptic and/or postsynaptic mechanism, is involved in the changes in synaptic transmission at the late phase.

\section{Oligodendrocyte depolarization/hyperpolarization modulates the induction of LTP at destination synapses}

As shown in Figure 4, the amplitude of EPSCs in bursting cells at the mid and distal subiculum increased at both the early and late phases after oligodendrocyte depolarization. Since the axonal conduction of multiple action potentials is synchronized in the early phase, which is supported by the observed decrease of CAP width in this period (Fig. 2C), it is possible that the induction of LTP, which shows associative and cooperative characteristics, is also modulated by oligodendrocyte depolarization when conditioning stimuli are applied in the early phase to induce LTP. We recorded EPSCs from bursting pyramidal cells in the mid and distal regions of the subiculum and delivered theta burst stimulation consisting of a different number of bursts $(5,10,15$, or 20 bursts) with each burst containing four pulses at $100 \mathrm{~Hz}$ and individual bursts separated by $200 \mathrm{~ms}$ (Fig. 7A). In the absence of
(Figure legend continued.) $\operatorname{mid}($ yellow, $n=7$ and $n=8$ ), and distal (red, $n=9$ and $n=11$ ) regions of the subiculum, expressed as a percentage of the mean EPSC amplitude during the 8 min period before light pulse delivery. The blue bars indicate the timing of blue light illumination $(500 \mathrm{~ms})$. The black bars indicate the measurement periods in (E). Insets are typical examples of a biocytin-stained pyramidal cell (left) and its firing pattern (right) in each group. Scale bar, $200 \mu \mathrm{m}$. E, Summary histogram for the changes in EPSC amplitude induced by oligodendrocyte depolarization at $1-3 \mathrm{~min}$ (left) and $28-30 \mathrm{~min}$ (right) after photostimulation. ${ }^{*} p<0.05$. 
A

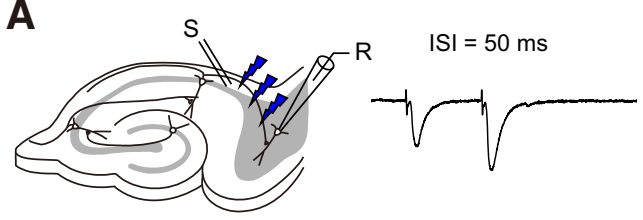

B

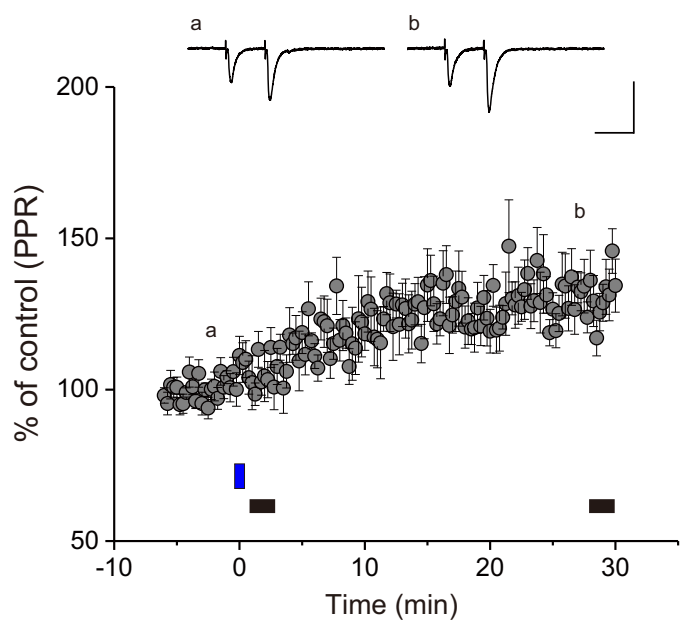

C
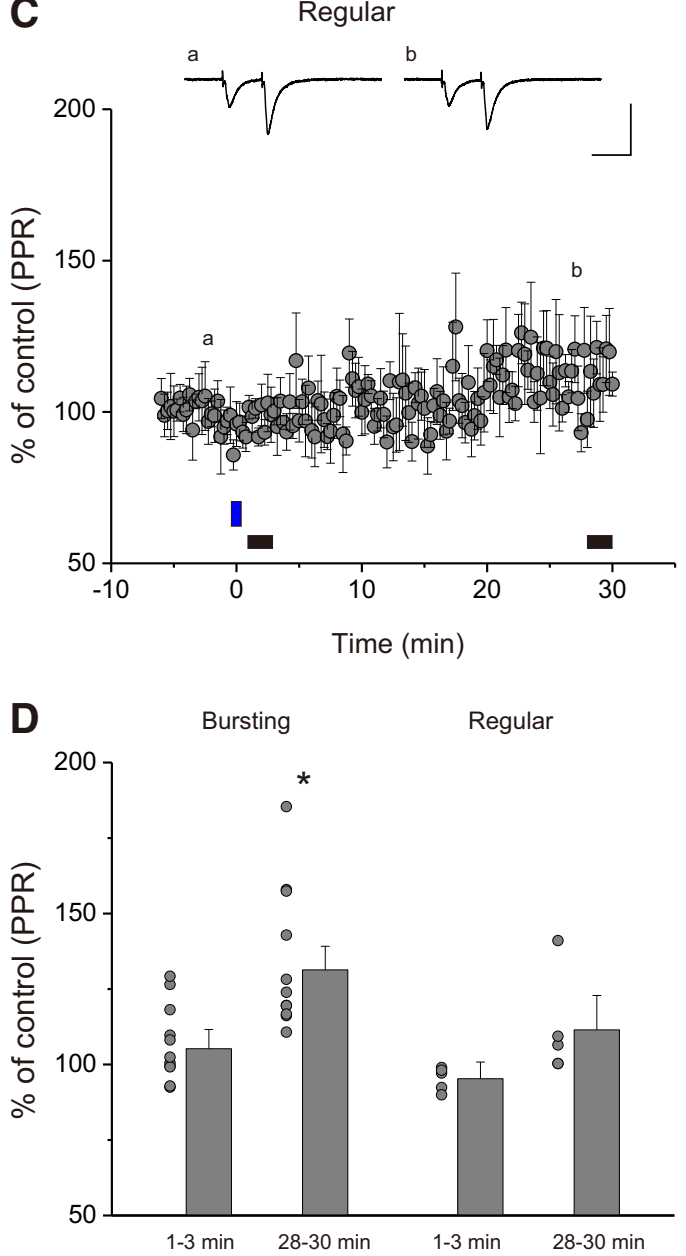

Figure 6. Effects of oligodendrocyte depolarization on the PPR of EPSCs at destination synapses. A, Schematic drawing showing the stimulating (S) and recording (R) electrodes for recording EPSCs from subicular neurons (left) and a typical EPSC waveform (right) with photostimulation, significant LTP was induced by the application of 20 bursts (35-40 min after theta burst stimulation: $146.9 \pm 6.6 \%$ of baseline; $n=7, \mathrm{t}_{10}=5.38, p<0.001$ vs no bursts), but not by 5,10 , or 15 bursts (Fig. $7 B-D$ ), indicating that the threshold for LTP induction by theta burst stimulation was between 15 and 20 bursts. In the photostimulation experiments, theta burst stimulation was delivered at 3 min after the end of photostimulation (Fig. 8A). Similar to the data presented in Figure 4, EPSC amplitude was increased after illumination with blue light $(500 \mathrm{~ms}$; Fig. $8 \mathrm{~B}, \mathrm{C})$. The delivery of both 15 and 20 bursts induced significant LTP [35-40 min after theta burst stimulation (i.e., 38-43 min after photostimulation): 15 bursts: $159.9 \pm$ $10.8 \%$ of baseline; $n=9, t_{(13)}=3.24, p=0.0064$ vs photostimulation without bursts; 20 bursts: $170.6 \pm 12.2 \%$ of baseline, $n=$ $13, t_{(17)}=2.97, p=0.0086$ vs photostimulation without bursts], while the delivery of either 5 or 10 bursts failed to induce LTP (Fig. $8 B-D$ ). These results show that the threshold for LTP induction is decreased by the effects of oligodendrocyte depolarization at destination synapses. It is well known that, during a series of theta burst stimulations, the initial burst increases the excitability of postsynaptic neurons, resulting in an increase of the sensitivity of postsynaptic neurons to subsequent bursts, by which LTP is more likely to be induced (Larson and Munkácsy, 2015). In addition to the effective properties of theta burst stimulation to induce LTP, it is possible that theta burst-induced LTP is facilitated by optogenetic oligodendrocyte depolarization, which synchronizes axonal conduction. On the basis of this hypothesis, we predicted that a reduction of oligodendrocyte depolarization during theta burst stimulation would have a negative effect on LTP induction. To examine this, we expressed ArchT on oligodendrocytes to reduce depolarization during theta burst stimulation (Fig. 9A). Unexpectedly, we found that PLP-ArchT mice, which were fed normal chow, exhibited a shivering phenotype at 3 weeks of age. TEM analysis demonstrated that PLP-ArchT mice showed severe hypomyelination (Fig. 9A), suggesting a toxic effect of ArchT on myelination. To avoid this possible effect, we administered Dox to PLP-ArchT mice from birth. Dox chow was changed to normal chow at 4 weeks of age, and the mice were killed at 6 weeks of age (Fig. 9B). TEM analysis demonstrated that the number of myelinated fibers, axon diameter, myelin thickness, and g-ratio in PLP-ArchT mice $(n=4)$ were comparable to those in control mice $\left(n=4\right.$; myelinated axon number: $t_{(6)}=$ 1.03, $p=0.34$; axon diameter: $t_{(6)}=0.41, p=0.70$; myelin thickness: $t_{(6)}=0.17, p=0.88$; g-ratio: $t_{(6)}=0.35, p=0.74$; Fig. $9 B-F)$. In the 2 week ArchT induction regimen, the mice did not show that the shivering phenotype and EGFP-tagged ArchT expression was observed in the white matter. Immunohistochemical analysis demonstrated that EGFP-positive fibrous structures were colabeled with PLP in PLP-ArchT mice, but that none of these structures was colabeled with NeuN, GFAP, or Ibal in the hippocampal alveus (Fig. 9G). We applied a 2 week Dox-off regimen to induce ArchT-EGFP expression after myelination. Yellow light illumination hyperpolarized currentclamped oligodendrocytes in PLP-ArchT mice $(9.5 \pm 1.4 \mathrm{mV}$,

$\leftarrow$

paired-pulse stimulation. $B, C$, Time course of the changes in the PPR of EPSCs induced by oligodendrocyte depolarization in bursting neurons $(\boldsymbol{B})$ and regular firing neurons $(\boldsymbol{C})$, expressed as a percentage of the mean EPSC amplitude during the 6 min period before light pulse delivery. Insets are typical waveforms evoked by paired stimuli obtained at the times ( $a$ and $b$ ) indicated in the main panel. Calibration: $50 \mathrm{~ms}, 200 \mathrm{pA}$. The black bars indicate the measurement periods in (D). D, Summary histogram for the changes in the PPR of EPSCs induced by oligodendrocyte depolarization at $1-3$ and $28-30 \mathrm{~min}$ after photostimulation. ${ }^{*} p<0.05$. 
A No photostim

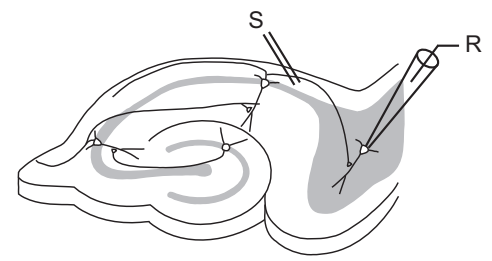

B
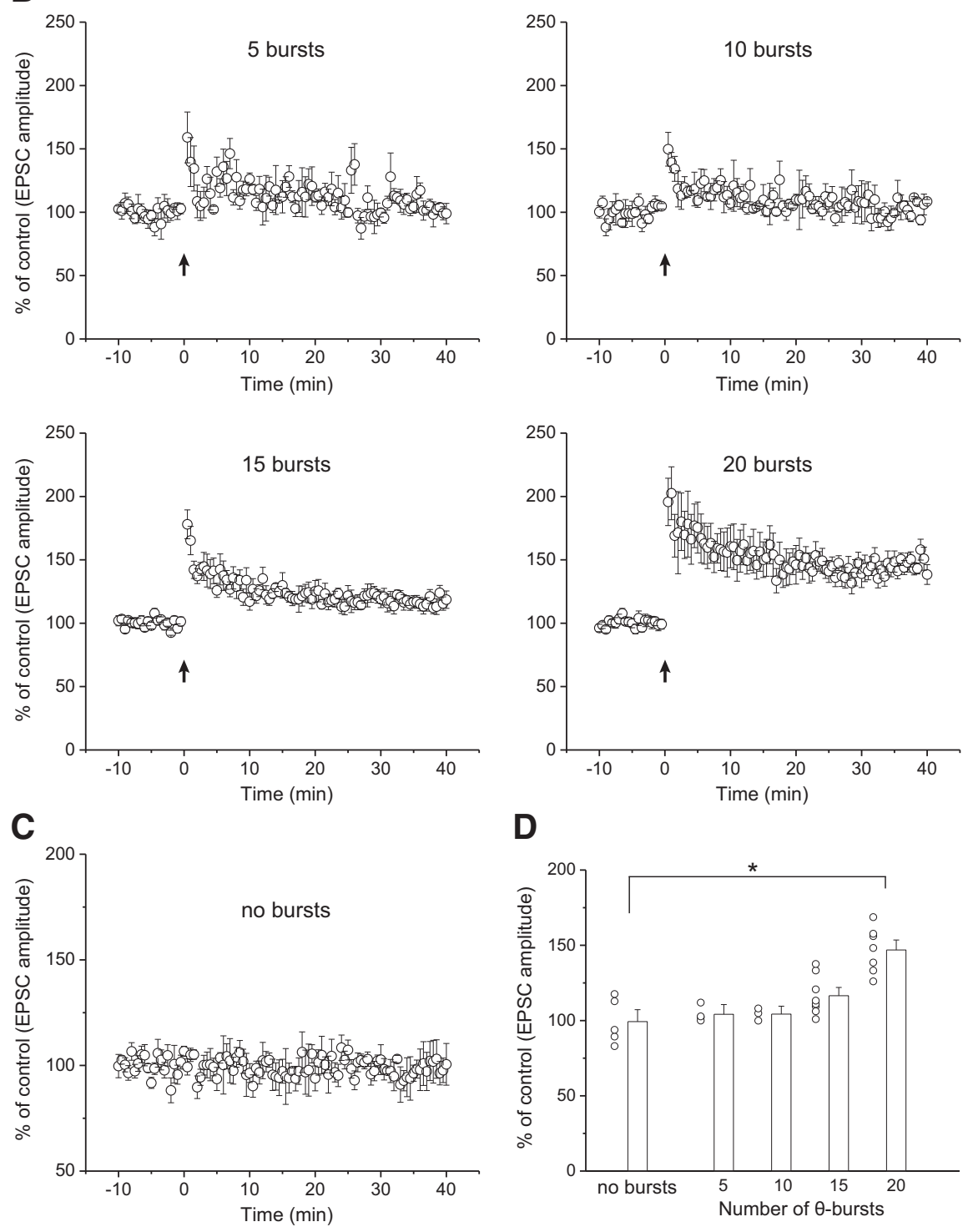

Figure 7. Induction of LTP at CA1-subicular bursting neuron synapses. $A$, Schematic drawing showing the stimulating $(S)$ and recording $(\mathrm{R})$ electrodes for recording EPSCS from subicular bursting neurons (left) and electrical stimulation protocol of theta burst stimulation for the induction of LTP (right). $\boldsymbol{B}$, Changes in EPSC amplitude induced by theta burst stimulation with different numbers of bursts $(5,10,15$, and 20 bursts), expressed as a percentage of the mean CAP amplitude during the 10 min period before theta burst stimulation in hippocampal slices from mice expressing $\mathrm{ChR2}$ on oligodendrocytes in the absence of photostimulation (5 bursts, $n=4 ; 10$ bursts, $n=3 ; 15$ bursts, $n=8 ; 20$ bursts, $n=7$ ). C, Time course of EPSC amplitude in the absence of light pulse delivery without theta burst stimulation $(n=5)$ for the data of control EPSCS. D, Summary histograms for the change in EPSC amplitude at 35-40 min after theta burst stimulation expressed as a percentage of the mean EPSC amplitude during the $10 \mathrm{~min}$ period before theta burst stimulation delivery. ${ }^{*} p<0.05$.

$n=5)$, but not in mice with the same genotype in which PLPArchT expression was blocked by Dox treatment $(0.16 \pm 0.14$ $\mathrm{mV}$ shift, $n=5)$, demonstrating the expression of functional ArchT (Fig. 9H). Input-output curves generated by plotting the amplitude of CAPs versus stimulation strength were not diffe- rent between wild-type mice $(n=4)$ and PLP-ArchT mice $\left(n=4 ; F_{(1,60)}=3.08\right.$, $p=0.13$, two-way repeated-measures ANOVA; Fig. 9I). Since oligodendrocytes are depolarized by theta burst stimulation with a duration of several tens of seconds (Yamazaki et al., 2007), we applied yellow light illumination for $30 \mathrm{~s}$ to reduce their depolarization. Oligodendrocyte hyperpolarization (30 s) showed no significant changes in the amplitude of CAPs recorded from the alveus (Fig. 10A) and the amplitude of EPSCs recorded from subicular bursting neurons (Fig. 10B), indicating that hyperpolarization had no effect on basic axonal conduction and synaptic transmission at destination synapses. Then, we delivered 15 or 20 bursts during yellow light illumination. Yellow light illumination was initiated at $5 \mathrm{~s}$ before the first burst stimulation and was terminated at $25 \mathrm{~s}$ after the end of the last (15th or 20th) burst to suppress oligodendrocyte depolarization induced by theta burst stimulation (Fig. 10C). Similar to the PLP-ChR2 mice (Fig. 7), in the absence of yellow light illumination, 15 bursts did not induce significant LTP (118.1 $\pm 7.5 \%$ of baseline, $n=7$ ), while 20 bursts induced significant LTP $(168.8 \pm 9.2 \%$ of baseline; $n=6$; Fig. 10C) in ArchTexpressing oligodendrocytes. These results suggest that ArchT expression in oligodendrocytes itself had no effect on the induction of LTP. When the oligodendrocytes were hyperpolarized by yellow light illumination, the induction of LTP was significantly suppressed (two-way ANOVA: $F_{(1,19)}=9.95, p=0.0052$ vs no yellow light illumination), with the magnitude of LTP induced by 20 bursts being significantly smaller than in the absence of hyperpolarization $(137.6 \pm 10.3 \%$ of baseline; $n=5, p=0.013$, Tukey's post hoc test; Fig. 10C). The changes in EPSCs induced by 15 bursts under yellow light illumination showed no significant difference compared with those in the absence of yellow light illumination. We also confirmed that the suppressive effect on LTP was induced by oligodendrocyte hyperpolarization rather than the effects of photostimulation alone by performing control experiments in PLP-ArchT mice with Dox treatment in which the magnitude of LTP was larger than in PLP-ArchT mice with photostimulation (two-way ANOVA, $F_{(1,15)}=4.58, p=0.047$; Fig. 10C). Thus, the induction of LTP by theta burst stimulation was suppressed by oligodendrocyte hyperpolarization, which reduced the synchronization of axonal conduction induced by theta burstinduced depolarization. These results suggest that the synchronization of axonal conduction by oligodendrocyte depolarization 
A

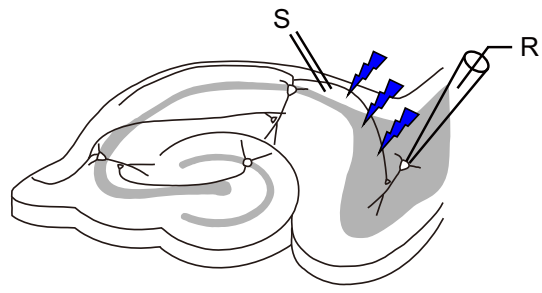

B
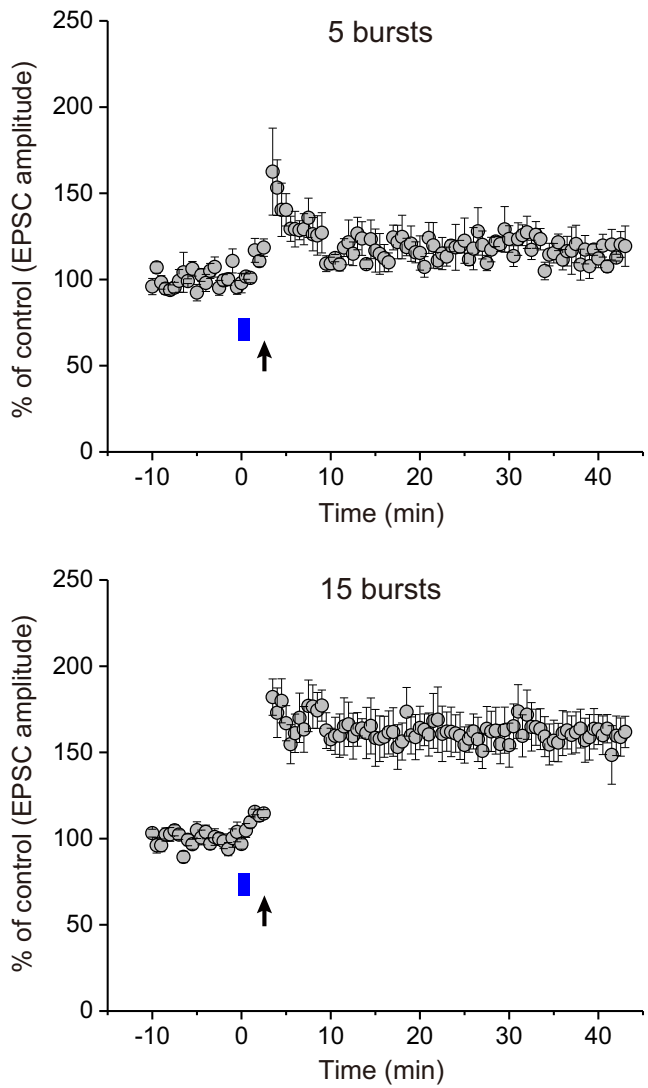

C

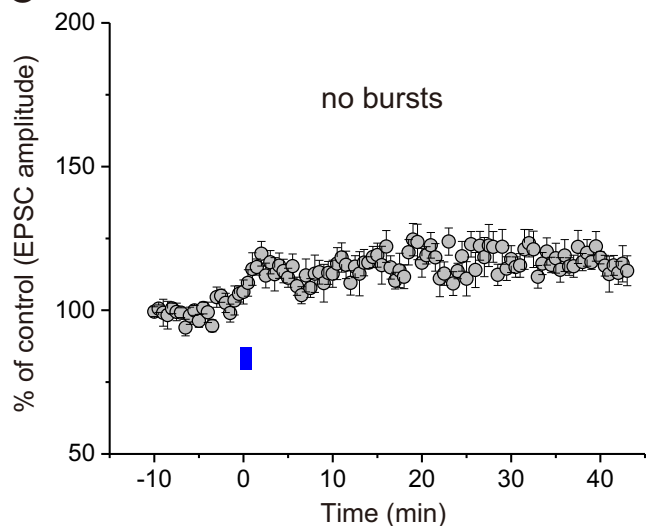

$\theta$-burst stimulation
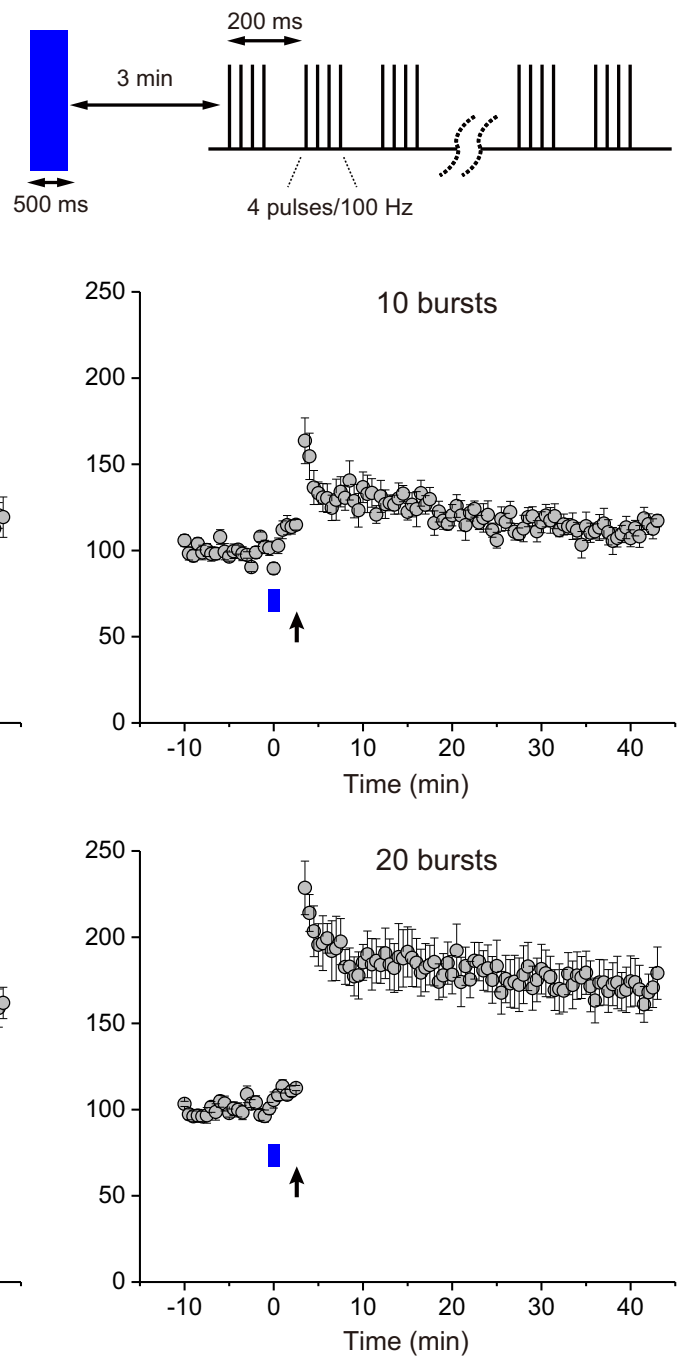

D

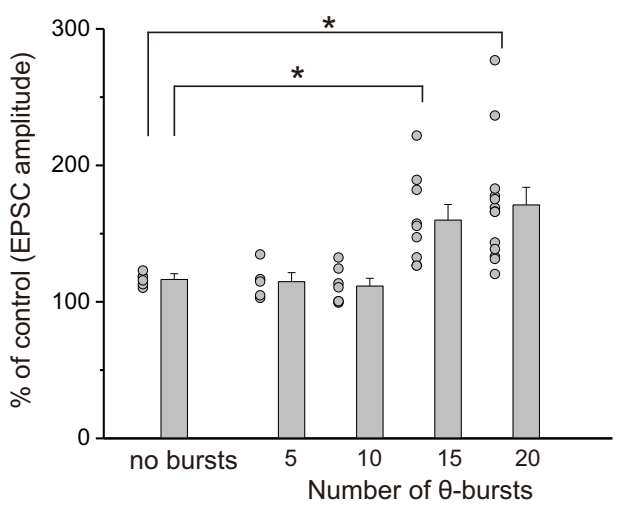

Figure 8. Effects of oligodendrocyte depolarization on LTP induction. $A$, Schematic drawing showing the stimulating $(S)$ and recording (R) electrodes for recording EPSCs from subicular neurons and blue light illumination (blue jagged arrow; left), and the protocol for photostimulation and electrical theta burst stimulation for the induction of LTP (right). $\boldsymbol{B}$, Changes in EPSC amplitude induced by theta burst stimulation with different numbers of bursts $(5,10,15$, and 20 bursts) in the presence of photostimulation ( 5 bursts, $n=5 ; 10$ bursts, $n=7 ; 15$ bursts, $n=9 ; 20$ bursts, $n=$ 13). Theta burst stimulation was delivered at 3 min after photostimulation, and EPSC amplitude was expressed as a percentage of the mean EPSC amplitude during the 10 min period before blue light illumination. C, Changes in EPSC amplitude induced by blue light illumination without theta burst stimulation $(n=6)$ expressed as a percentage of the mean EPSC amplitude during the 10 min period before light pulse delivery. D, Summary histograms for the change in EPSC amplitude at 35-40 min after theta burst stimulation (38-43 min after photostimulation) expressed as a percentage of the mean EPSC amplitude during the 10 min period before photostimulation. ${ }^{*} p<0.05$. 
A

PLP-tTA::tetO-ArchT, Dox off

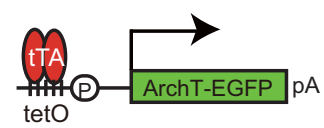

B

PLP-tTA::tetO-ArchT, Dox on to off 2 weeks

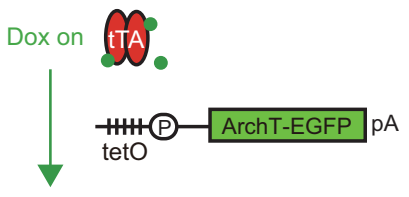

Dox off $2 \mathrm{~W}$

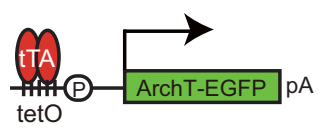

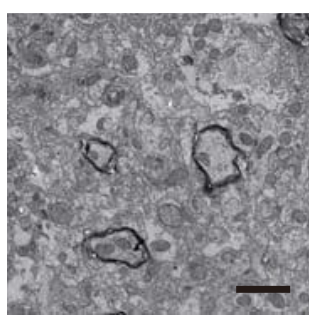

C

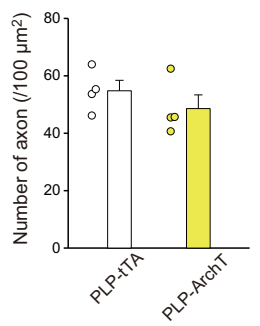

E

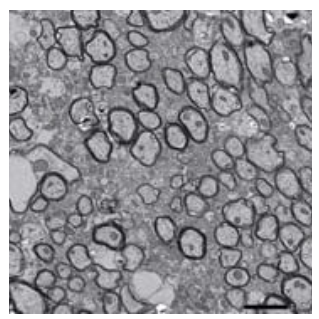

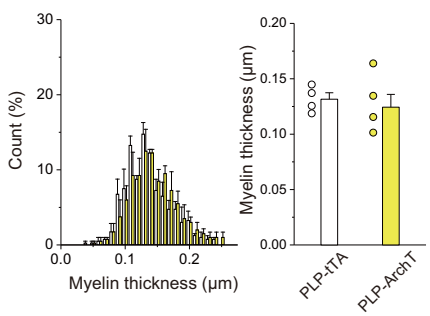

F

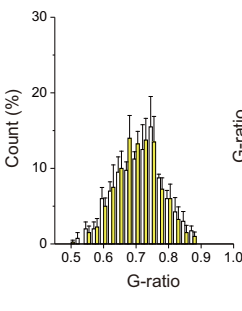

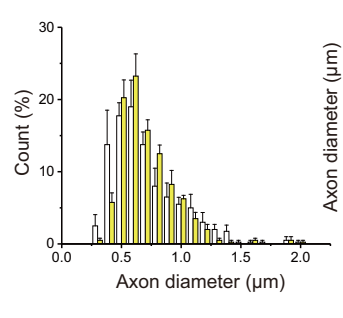
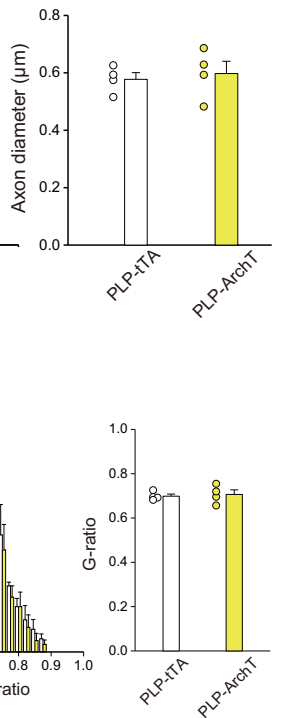

G
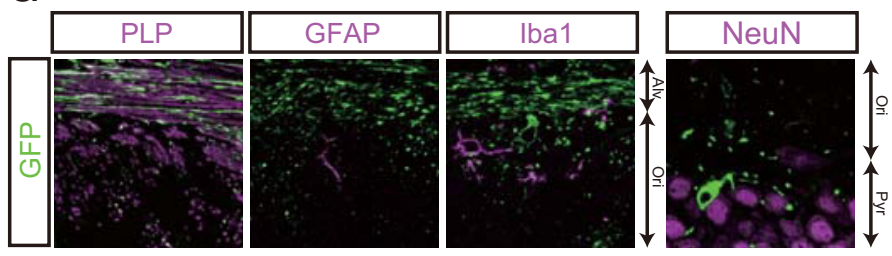

$\mathbf{H}$

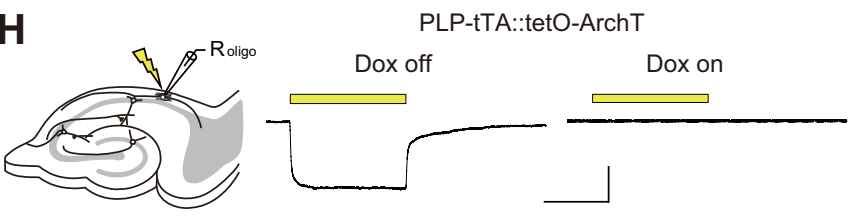

I

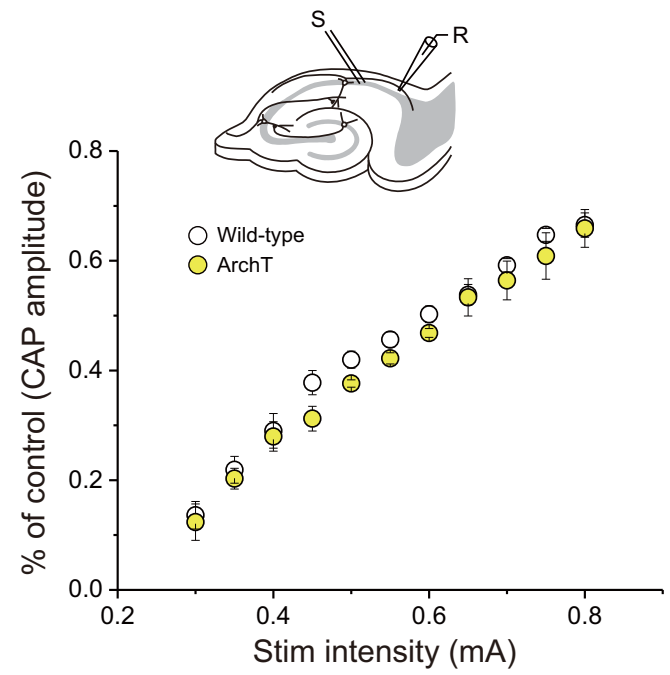

Figure 9. Characterization of PLP-ArchT mice. A, Induction of oligodendrocyte-specific ArchT-EGFP expression in the tet system (left). tTA, tetracycline-controlled transcriptional activator; tet0, tetracycline-controlled transcriptional activator-dependent promoter. Right, Transmission electron micrograph showing severe hypomyelination in the corpus callosum at 4 weeks of age without Dox treatment. Scale bar, $2 \mu \mathrm{m}$. B, Left, Induction of oligodendrocyte-specific ArchT-EGFP expression in the absence of Dox. ArchT-EGFP was induced for 2 weeks after the switch from Dox administration to no Dox. Right, Electron micrograph showing the normal appearance of myelin structure in the corpus callosum at 6 weeks of age. Scale bar, $2 \mu \mathrm{m}$. C, The number of myelinated axons in the corpus callosum of PLP-ArchT mice $(n=4)$ and control mice $(n=4)$. $\boldsymbol{D}-\boldsymbol{F}$, Histograms (left) and group data (right) of axon diameter $(\boldsymbol{D})$, myelin thickness $(\boldsymbol{E})$, and g-ratio $(\boldsymbol{F})$ of PLP-ArchT mice $(n=4)$ and control mice $(n=4)$. $\boldsymbol{G}$, Double immunohistochemistry showing that PLP-positive myelin fibers were labeled with GFP ( $37.7 \pm 1.8 \%, 4$ sections, 2 animals). None of the GFAP-, Iba1-, or NeuN-positive signals were colabeled with an anti-GFP antibody. Purple, Marker; green, GFP; Alv, alveus; Ori, stratum oriens; Pyr, CA1 pyramidal cell layer in the hippocampus. Scale bar, $10 \mu \mathrm{m}$. $\boldsymbol{H}$, Schematicdrawing showing the recording (R) electrode for whole-cell recordings from oligodendrocytes in the alveus (left). Typical responses from a current-clamped oligodendrocyte caused by yellow light photostimulation applied at the time indicated by the yellow bar in PLP-ArchT mice (middle) and in mice with the same genotype as PLP-ArchT mice, but expression was blocked by Doxtreatment (right). Calibration:5 ms, $5 \mathrm{mV}$. I, Top, Schematic drawing showing the stimulating (S) and Relectrodes for recording CAPs. Bottom, Input- output relationships of CAP amplitude in wild-type and PLP-ArchT mice.

during theta burst stimulation contributes to the effective induction of LTP induced by theta burst stimulation.

\section{Discussion}

\section{Region- and cell type-specific modulation of synaptic} transmission at destination synapses

The modulatory effects of the depolarization of oligodendrocytes on synaptic responses between the presynaptic terminals of axons myelinated by depolarized oligodendrocytes and postsynaptic neurons showing that burst firing depends on the region of the subiculum in which they are located (Fig. 4). This region dependency was related to whether the facilitative effects of oligo- dendrocyte depolarization on axonal conduction were observed in the axons projecting to each region of the subiculum (Fig. 5). Therefore, it is possible that the axons terminating in each region have different conduction properties. In fact, the mean latency of the antidromic action potential, which was calculated by dividing latency by the distance between the tip of the stimulating electrode and the soma of the recorded cell (in microseconds per millimeter), for the axons of CA1 pyramidal cells close to the subiculum was $\sim 1.8$ times greater than that of the axons of CA1 pyramidal cells close to the CA2 region, indicating that the properties of myelin, such as the number of layers of the myelin sheath, internodal length, and capacitance, differ between these regions. These differences 
A
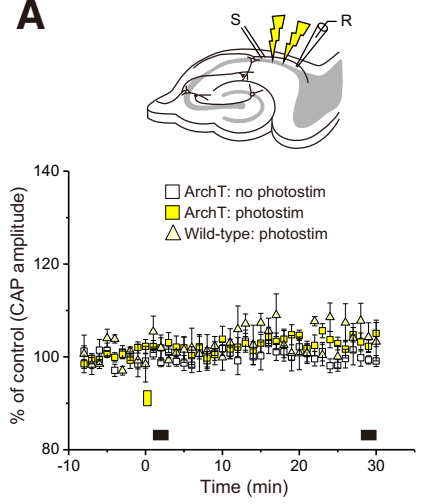

$\square$ ArchT: no photostim $\square$ ArchT: photostim

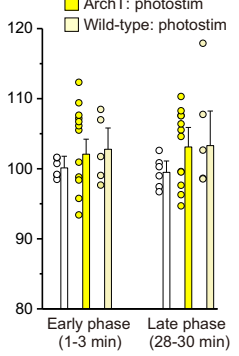

B
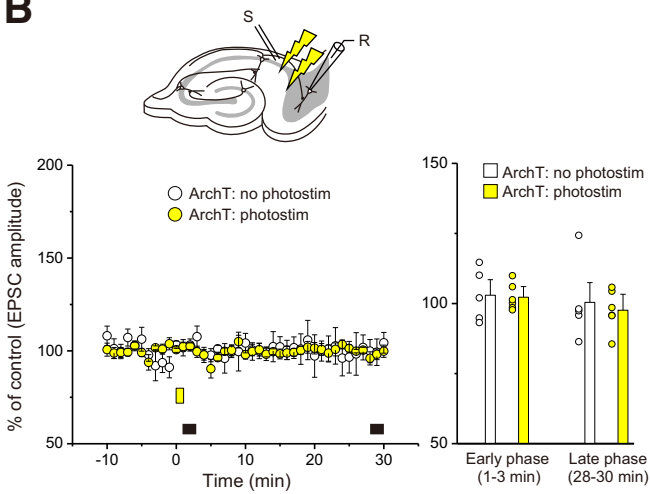

C
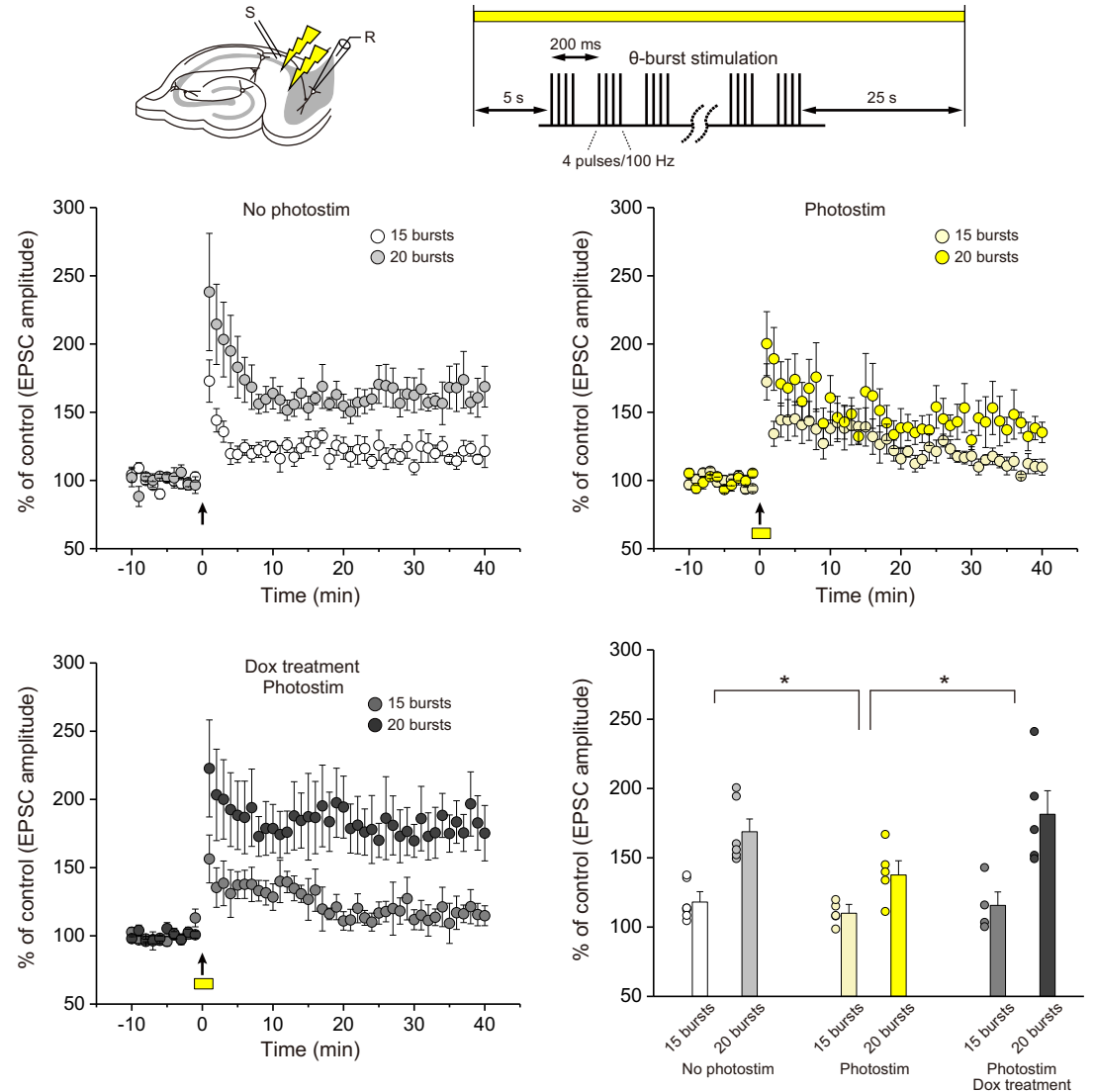

Figure 10. Effects of oligodendrocyte hyperpolarization on axonal conduction in the alveus and LTP induction at destination synapses. $A$, Top, Schematic drawing showing the stimulating ( $S$ ) and recording (R) electrodes for recording (APs. Bottom left, Changes in CAP amplitude induced by yellow light illumination for $30 \mathrm{~s}$, starting at 0 min (yellow bar), expressed as a percentage of the mean CAP amplitude during the 8 min period before light pulse delivery $(n=12)$. The white squares and light yellow triangles are the data without photostimulation in PLP-ArchT mice $(n=6)$ and with photostimulation in control wild-type mice $(n=5)$, respectively. Bottom right, Summary histograms for the change in CAP amplitude at $1-3$ or $28-30$ min, the periods are indicated by the black bars, with no stimulation or after photostimulation for the indicated time, expressed as a percentage of the mean CAP amplitude during the 8 min period before yellow light pulse delivery. $\boldsymbol{B}$, Top, Schematic drawing showing the stimulating $(S)$ and recording (R) electrodes for recording EPSCs from subicular bursting neurons. Bottom left, Changes in EPSC amplitude induced by yellow light illumination for $30 \mathrm{~s}$, starting at $0 \mathrm{~min}$ (yellow bar), expressed as a percentage of the mean EPSC amplitude during the $10 \mathrm{~min}$ period before light pulse delivery $(n=6)$. The white circles are the data without photostimulation $(n=5)$. Bottom right, Summary histograms for the change in EPSC amplitude at 1-3 or 28-30 min, the periods are indicated by the black bars, with no stimulation or after photostimulation for the indicated time, expressed as a percentage of the mean EPSC amplitude during the 10 min period before yellow light pulse delivery. C, Schematic drawing showing the stimulating (S) and recording (R) electrodes for recording EPSCs from subicular neurons and yellow light illumination (yellow jagged arrow; top left), and the protocol for photostimulation and electrical theta burst stimulation for the induction of LTP (top right). Changes in EPSC amplitude induced by theta burst stimulation ( 15 or 20 bursts) in the absence ( 15 bursts, $n=7 ; 20$ bursts, $n=$ 6; middle left) and presence ( 15 bursts, $n=5 ; 20$ bursts, $n=5$; middle right) of yellow light illumination expressed as a percentage of the mean EPSC amplitude during the 10 min period before theta burst stimulation. Yellow light illumination was initiated at $5 \mathrm{~s}$ before first burst stimulation and terminated at $25 \mathrm{~s}$ after the end of the last (15th or 20th) burst, as indicated by the yellow bar. Bottom, Left, Changes in EPSC amplitude induced by theta burst stimulation in the presence (15 bursts, $n=4 ; 20$ bursts, $n=5$ ) of yellow light illumination in PLP-ArchT mice with Dox treatment. Bottom, Right, Summary histograms for the change in EPSC amplitude at 35-40 min after theta burst stimulation with or without photostimulation in PLP-ArchT mice or with photostimulation in PLP-ArchT mice with Dox treatment, expressed as a percentage of the mean EPSC amplitude during the 10 min period before yellow light pulse delivery. ${ }^{*} p<0.05$ (two-way ANOVA). 
in the myelin properties of axons projecting to different regions of the subiculum might result in the different effects of oligodendrocyte depolarization on axonal conduction, leading to the region-specific modulation of EPSCs at destination synapses. This region-specific modulation might contribute to the adjustment of the excitability of neuronal circuits as needed for circuit function in each region through interactions between individual axons and oligodendrocytes.

Currently, it is unknown why the effects of oligodendrocyte depolarization depend on the firing pattern of subicular pyramidal cells; however, the mechanism for LTP induction is different between cell types. In regular firing cells, LTP induction depends upon the activation of postsynaptic NMDA receptors and subsequent increase in $\mathrm{Ca}^{2+}$. Conversely, in bursting cells, presynaptic mechanisms account for LTP induction (Behr et al., 2009). This difference might be related to the cell-type specificity of the effects in some form.

When considering the function of myelin in the CNS, the term "oligodendrocyte-bound axonal bale" is used to refer to a group of axons myelinated by a given oligodendrocyte (de $\mathrm{Hoz}$ and Simons, 2015). Although it is unclear whether the axons in a bale are functionally correlated or simply located close to a bailing oligodendrocyte, since oligodendrocytes selectively myelinate a particular set of axons in white matter (Osanai et al., 2017), it is possible that axons synaptically connected to bursting cells and to regular firing cells are baled by different oligodendrocytes (Fig. 11). If so, the properties of oligodendrocytes baling each group of axons are different, and this might result in the cell-type specificity for the modulatory effects of oligodendrocyte depolarization. In that case, the difference in the electrophysiological properties of axons connected to each cell type should also be investigated. The spatial distribution of bursting cells is reportedly distinct from that of regular firing cells in the subiculum (Staff et al., 2000; Jarsky et al., 2008) and the projections of subicular neurons are topographic (Ishizuka, 2001). Thus, the cell type-specific modulation by oligodendrocyte depolarization might modulate the output from the hippocampus in a target-selective manner.

\section{Properties of the enhanced excitatory synaptic transmission induced by oligodendrocyte depolarization at destination synapses}

As demonstrated in Figure 4, EPSC amplitude in bursting cells at the mid and distal subiculum showed an immediate and lasting increase, for at least $30 \mathrm{~min}$, after oligodendrocyte depolarization. Although the magnitudes of the increase in EPSC amplitude at 1-3 and 28-30 min after photostimulation were similar, it is possible that these increases involve two different phases. Consistent with this hypothesis, the PPR of EPSCs showed only a gradual increase without an immediate increase after photostimulation. Since increased glutamate release from presynaptic terminals decreases the PPR, it is considered that, at the early phase in which the PPR was not changed by oligodendrocyte depolarization, a postsynaptic mechanism is responsible for the increased amplitude of EPSCs. However, since oligodendrocytes and myelin have no direct morphological relationship with postsynaptic components, it is unlikely that changes in postsynaptic glutamate receptors are involved in the increase in EPSC amplitude. Since axonal conduction is synchronized at the early phase, which was supported by the observation that CAP width was decreased and CAP area showed no significant change in this phase (Fig. $2 C, D$ ), the action potentials conducted by each axon would arrive simultaneously at the target postsynaptic neurons without any changes in glutamate release from each presynaptic terminal of the axons,

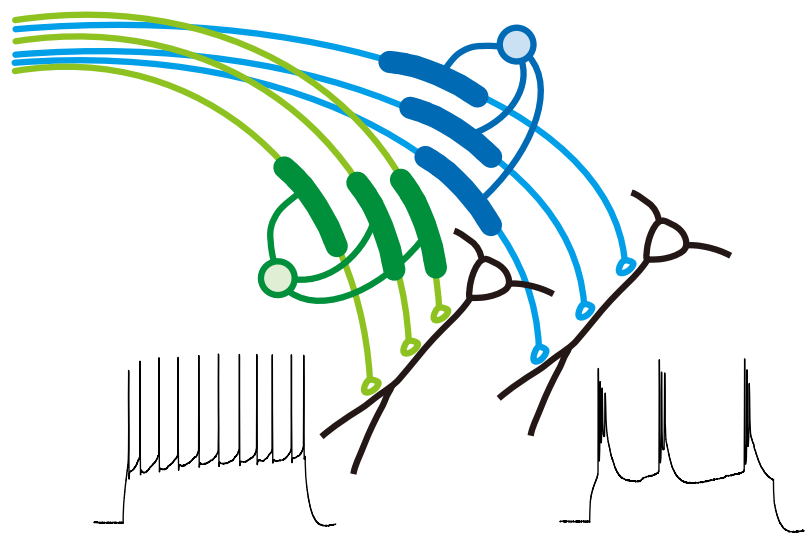

Figure 11. A schematic presentation of the possibility of distinct oligodendrocyte-bound axonal bales for connecting to bursting cells (blue) and regular firing cells (green).

resulting in an increase of temporal summation at postsynaptic sites, leading to the observed increase in EPSC amplitude.

With regard to the late phase, both the EPSC amplitude and the PPR of EPSCs were increased. Since axonal conduction is not synchronized at the late phase (Fig. 2C), the increase of temporal summation at postsynaptic sites is not involved in these changes in EPSCs and the PPR. In a previous study, it was speculated that the facilitation of axonal conduction at the late phase involves an increase in the number of firing axons and/or an increase in the amplitude and/or duration of each action potential conducted along each axon (Yamazaki et al., 2014). Since an increase in the amplitude and/or duration of each action potential would increase glutamate release from each axon, which leads to a decrease of the PPR, it is unlikely that changes in the kinetics of each action potential are involved in the changes at the late phase. Thus, an increase in the number of axons activated by electrical stimulation at a given intensity due to a decrease in the firing threshold, including those axons that had not generated action potentials previously, resulting in an increased number of activated axons connecting to recorded subicular neurons, could be the underlying mechanism for the changes at the late phase. With regard to the increase in the PPR at the late phase, the involvement of ephaptic interactions and their modulation is suggested. An ephapse is a point of contact where two or more nerve cell processes touch without forming a typical synaptic contact, and some form of transmission may occur at such nonsynaptic contact sites. Although ephaptic interactions are mainly observed between unmyelinated axons, it is possible that similar interactions occur in myelinated fibers (Debanne et al., 2011). In the paired-pulse stimulation experiment, ephaptic interactions could occur as follows: action potentials evoked by the first stimulation have minor electrical effects on other axons, thereby increasing axonal excitability; and the number of axons activated by the second stimulation at a given intensity, which is the same as that of the initial stimulation. Oligodendrocyte depolarization might enhance these ephaptic interactions, which would lead to a greater increase in the number of activated axons, resulting in the observed increase of the PPR of EPSCs at the late phase.

Since the extent of oligodendrocyte depolarization is dependent on how many neurons are excited and since oligodendrocytes can show long-lasting depolarization in response to axonal activity, a trace of the activation of neuronal circuits might be kept as oligodendrocyte depolarization, which then makes the neuronal circuits that depolarized the oligodendrocytes more active. 


\section{Enhancement of LTP induction by oligodendrocyte depolarization at destination synapses}

On the relationship between oligodendrocyte lineage cells and LTP, Sakry et al. (2014) demonstrated that these cells modulate signaling via glutamate receptors in neuronal synapses within the somatosensory cortex, including NMDA receptor-dependent LTP, by activity-dependent ectodomain shedding of NG2. This provides direct evidence that oligodendrocyte precursor cells are functionally integrated in the neuronal network and modulate glutamatergic synapses in the adult brain. However, a few reports have described a role for mature myelinating oligodendrocytes in LTP induction. During theta burst stimulation, oligodendrocytes are depolarized by the initial bursts, which activates NMDA receptors and voltage-dependent channels expressed on oligodendrocytes. Since the intracellular volume of the myelinating processes of oligodendrocytes is small, ion influx through these receptors and channels causes a significant increase in ionic concentration, leading to osmotic water influx. The increased volume of myelinating processes caused by osmotic water influx increases the insulation of the axon, enabling more rapid conduction of action potentials (Yamazaki et al., 2010). These changes result in the synchronization of multiple action potentials in the subsequent bursts, which effectively activates the mechanism for LTP induction. Optogenetic depolarization of oligodendrocytes facilitated the coordination of axonal conduction through additional depolarization and enhanced LTP induction. The suppressive effects of oligodendrocyte hyperpolarization by the activation of ArchT (Fig. 10) support this idea. Since the oligodendrocyte hyperpolarization inhibits the activation of NMDA receptors and voltage-dependent channels during theta burst stimulation, the mechanism for LTP induction involving oligodendrocyte depolarization would be suppressed.

In addition to synchronization by oligodendrocyte depolarization, it is possible that a larger number of axons, which do not fire in the test stimuli, are recruited during theta burst stimulation. This increase in the number of axons, which is enhanced by optogenetic oligodendrocyte depolarization, might lower the threshold for LTP induction, and it might have some relationship with the mechanism of the enhancement of CAP during the late phase, which is due to a decrease in the firing threshold induced by oligodendrocyte depolarization. It is reported that, differently from the induction of LTP at Schaffer collateral-CA1 synapses, LTP at the synapses between CA1 neurons and subicular bursting neurons is mainly induced by a presynaptic mechanism (Behr et al., 2009). The activation of PKA by a calcium-dependent adenylyl cyclase (Wozny et al., 2008a) or the activation of presynaptic NMDA receptors (especially NR2B-containing receptors; Wozny et al., 2008b; Behr et al., 2009) is involved in this presynaptic mechanism. The properly timed activation of presynaptic nicotinic receptors enhances the induction of LTP (Ji et al., 2001). Similarly, synchronized axonal conduction along the axons of CA1 pyramidal cells, which is enhanced by optogenetic oligodendrocyte depolarization in addition to theta burst stimulation, would activate presynaptic NMDA receptors in a timely and effective manner and then increase the presynaptic mechanism for LTP induction. Since the subiculum serves as the major relay station for the unidirectional outflow of information from the hippocampus, the observed facilitation of synaptic transmission and LTP induction by oligodendrocyte depolarization in the subiculum may influence the transfer of information from the hippocampus to subcortical and cortical brain areas.

\section{References}

Amaral DG, Dolorfo C, Alvarez-Royo P (1991) Organization of CA1 projections to the subiculum: a PHA-L analysis in the rat. Hippocampus 1:415-435.

Behr J, Wozny C, Fidzinski P, Schmitz D (2009) Synaptic plasticity in the subiculum. Prog Neurobiol 89:334-342.

Berger T, Walz W, Schnitzer J, Kettenmann H (1992) GABA- and glutamate- activated currents in glial cells of the mouse corpus callosum slice. J Neurosci Res 31:21-27.

Debanne D, Campanac E, Bialowas A, Carlier E, Alcaraz G (2011) Axon physiology. Physiol Rev 91:555-602.

de Hoz L, Simons M (2015) The emerging functions of oligodendrocytes in regulating neuronal network behavior. Bioessays 37:60-69.

Fields RD (2010) Change in the brain's white matter. Science 330:768-769.

Inamura N, Sugio S, Macklin WB, Tomita K, Tanaka KF, Ikenaka K (2012) Gene induction in mature oligodendrocytes with a PLP-tTA mouse line. Genesis 50:424-428.

Ishizuka N (2001) Laminar organization of the pyramidal cell layer of the subiculum in the rat. J Comp Neurol 435:89-110.

Jarsky T, Mady R, Kennedy B, Spruston N (2008) Distribution of bursting neurons in the CAl region and the subiculum of the rat hippocampus. J Comp Neurol 506:535-547.

Ji D, Lape R, Dani JA (2001) Timing and location of nicotinic activity enhances or depresses hippocampal synaptic plasticity. Neuron 31:131-141.

Káradóttir R, Cavelier P, Bergersen LH, Attwell D (2005) NMDA receptors are expressed in oligodendrocytes and activated in ischaemia. Nature 438:1162-1166.

Larson J, Munkácsy E (2015) Theta-burst LTP. Brain Res 1621:38-50.

Osanai Y, Shimizu T, Mori T, Yoshimura Y, Hatanaka N, Nambu A, Kimori Y, Koyama S, Kobayashi K, Ikenaka K (2017) Rabies virus-mediated oligodendrocyte labeling reveals a single oligodendrocyte myelinates axons from distinct brain regions. Glia 65:93-105.

Sakry D, Neitz A, Singh J, Frischknecht R, Marongiu D, Binamé F, Perera SS, Endres K, Lutz B, Radyushkin K, Trotter J, Mittmann T (2014) Oligodendrocyte precursor cells modulate the neuronal network by activitydependent ectodomain cleavage of glial NG2. PLoS Biol 12:e1001993.

Shibata S, Murota Y, Nishimoto Y, Yoshimura M, Nagai T, Okano H, Siomi MC (2015) Immuno-electron microscopy and electron microscopic in situ hybridization for visualizing piRNA biogenesis bodies in drosophila ovaries. Methods Mol Biol 1328:163-178.

Staff NP, Jung HY, Thiagarajan T, Yao M, Spruston N (2000) Resting and active properties of pyramidal neurons in subiculum and CA1 of rat hippocampus. J Neurophysiol 84:2398-2408.

Tanaka KF, Matsui K, Sasaki T, Sano H, Sugio S, Fan K, Hen R, Nakai J, Yanagawa Y, Hasuwa H, Okabe M, Deisseroth K, Ikenaka K, Yamanaka A (2012) Expanding the repertoire of optogenetically targeted cells with an enhanced gene expression system. Cell Rep 2:397-406.

Tsunematsu T, Tabuchi S, Tanaka KF, Boyden ES, Tominaga M, Yamanaka A (2013) Long-lasting silencing of orexin/hypocretin neurons using archaerhodopsin induces slow-wave sleep in mice. Behav Brain Res 255:64-74.

Wozny C, Maier N, Fidzinski P, Breustedt J, Behr J, Schmitz D (2008a) Differential cAMP signaling at hippocampal output synapses. J Neurosci 28:14358-14362.

Wozny C, Maier N, Schmitz D, Behr J (2008b) Two different forms of longterm potentiation at CA1-subiculum synapses. J Physiol 586:2725-2734.

Yamazaki Y, Hozumi Y, Kaneko K, Sugihara T, Fujii S, Goto K, Kato H (2007) Modulatory effects of oligodendrocytes on the conduction velocity of action potentials along axons in the alveus of the rat hippocampal CA1 region. Neuron Glia Biol 3:325-334.

Yamazaki Y, Hozumi Y, Kaneko K, Fujii S, Goto K, Kato H (2010) Oligodendrocytes: facilitating axonal conduction by more than myelination. Neuroscientist 16:11-18.

Yamazaki Y, Fujiwara H, Kaneko K, Hozumi Y, Xu M, Ikenaka K, Fujii S, Tanaka KF (2014) Short- and long-term functional plasticity of white matter induced by oligodendrocyte depolarization in the hippocampus. Glia 62:1299-1312.

Ziak D, Chvátal A, Syková E (1998) Glutamate-, kainate- and NMDAevoked membrane currents in identified glial cells in rat spinal cord slice. Physiol Res 47:365-375. 